\title{
The cGAS-STING pathway drives type IIFN immunopathology in COVID-19
}

https://doi.org/10.1038/s41586-022-04421-w

Received: 18 June 2021

Accepted: 13 January 2022

Published online: 19 January 2022

Open access

Check for updates

\begin{abstract}
Jeremy Di Domizio, ${ }^{1,9}$, Muhammet F. Gulen ${ }^{2,9}$, Fanny Saidoune ${ }^{1,9}$, Vivek V. Thacker ${ }^{2,9}$, Ahmad Yatim ${ }^{1,9}$, Kunal Sharma ${ }^{2}$, Théo Nass ${ }^{2}$, Emmanuella Guenova', Martin Schaller ${ }^{3}$, Curdin Conrad', Christine Goepfert ${ }^{4,5}$, Laurence de Leval ${ }^{6}$, Christophe von Garnier ${ }^{7}$, Sabina Berezowska ${ }^{6}$, Anaëlle Dubois ${ }^{8}$, Michel Gilliet ${ }^{1,10} \otimes$ \& Andrea Ablasser ${ }^{2,10 凶}$
\end{abstract}

COVID-19, which is caused by infection with SARS-CoV-2, is characterized by lung pathology and extrapulmonary complications ${ }^{1,2}$. Type I interferons (IFNs) have an essential role in the pathogenesis of COVID-19 $\left(\mathrm{refs}^{3-5}\right)$. Although rapid induction of type IIFNs limits virus propagation, a sustained increase in the levels of type I IFNs in the late phase of the infection is associated with aberrant inflammation and poor clinical outcome ${ }^{5-17}$. Here we show that the cyclic GMP-AMP synthase (cGAS)stimulator of interferon genes (STING) pathway, which controls immunity to cytosolic DNA, is a critical driver of aberrant type IIFN responses in COVID-19 (ref. ${ }^{18}$ ). Profiling COVID-19 skin manifestations, we uncover a STING-dependent type IIFN signature that is primarily mediated by macrophages adjacent to areas of endothelial cell damage. Moreover, cGAS-STING activity was detected in lung samples from patients with COVID-19 with prominent tissue destruction, and was associated with type IIFN responses. A lung-on-chip model revealed that, in addition to macrophages, infection with SARS-CoV-2 activates cGAS-STING signalling in endothelial cells through mitochondrial DNA release, which leads to cell death and type I IFN production. In mice, pharmacological inhibition of STING reduces severe lung inflammation induced by SARS-CoV-2 and improves disease outcome. Collectively, our study establishes a mechanistic basis of pathological type IIFN responses in COVID-19 and reveals a principle for the development of host-directed therapeutics.
To obtain insight into aberrant immunological processes at the tis-

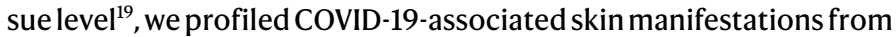
10 hospitalized patients with moderate-to-severe COVID-19 disease and compared the resultant signatures with those obtained from skin lesions of patients with inflammatory skin disease ${ }^{20}$ (Extended Data Fig.1). Transcriptome analysis revealed that COVID-19 profiles clustered with profiles from cutaneous lupus erythematosus (CLE), but separated from signatures of other skin diseases, such as psoriasis, atopic dermatitis and lichen planus (Fig. 1a, Extended Data Fig. 2). Transcriptional similarities between COVID-19 and CLE samples were based on the expression of IFNs (IFNA2, IFNA4, IFNA1, IFNA13, IFNB1, IFNL2 and IFNL3) in purpuro-necrotic COVID-19 skin lesions and IFN-stimulated genes (ISGs) (IFIT2, BST2, IRF7, OASL, MX1, IFITM1, IFIT2, IFI35, IFIH1, ISG15, CXCL1O and CXCL9) in those with a maculo-papular phenotype (Fig. 1a). Notably, both COVID-19 skin phenotypes-but not CLEshowed a marked upregulation of genes related to macrophage function, including macrophage receptors (CD209, CLU, MARCO, FCGR2A, CLEC5A, CD163, MRC1 and BST1), differentiation factors (IL32) and monocyte-recruiting chemokines (CXCL2 and CCL2) (Fig. 1a, Extended
Data Fig. 2). Pro-inflammatory cytokines ( $T N F, I L 6, I L 1 B$ and IL1A) were also induced in COVID-19 skin biopsies (Fig. 1a). The immune correlates detected in skin lesions of patients with moderate-to-severe COVID-19 therefore resemble those reported for the lung ${ }^{8,17}$, suggesting a shared mechanism of immunopathology across different organs.

\section{Vascular damage and type I IFNs in skin lesions}

Next, we comparatively analysed the immune cell composition in skin lesions of patients with COVID-19 and patients with CLE by immunostaining. Consistent with the specific expression of macrophage signature genes, the numbers of $\mathrm{CD}_{163}{ }^{+}$macrophages were higher in COVID-19 skin lesions relative to CLE (Fig. 1b, Extended Data Fig. 3a). By contrast, plasmacytoid dendritic cells-but not macrophages-were enriched in CLE samples, whereas the numbers of neutrophils and T cells were similar in the two conditions (Fig. 1b, Extended Data Fig. 3a). Further examination of macrophages revealed that these cells consistently showed a robust IFN $\beta$ response across all samples (Fig. 1c, d, f). Endothelial cells and other cell types also showed a marked IFN $\beta$ signal,

1Department of Dermatology, CHUV University Hospital and University of Lausanne (UNIL), Lausanne, Switzerland. ${ }^{2}$ Global Health Institute, Swiss Federal Institute of Technology Lausanne

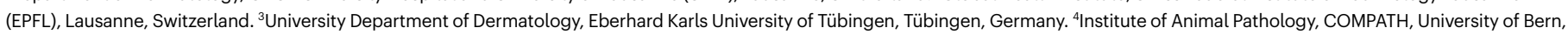
Bern, Switzerland. ${ }^{5} \mathrm{H}$ istology Core Facility, Swiss Federal Institute of Technology Lausanne (EPFL), Lausanne, Switzerland. ${ }^{6}$ Institute of Pathology, CHUV University Hospital and University of

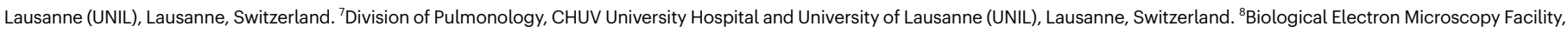

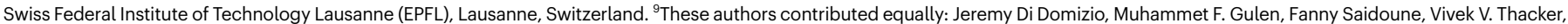
Ahmad Yatim. ${ }^{10}$ These authors jointly supervised this work: Michel Gilliet, Andrea Ablasser. ${ }^{\circledR}$ e-mail: michel.gilliet@chuv.ch; andrea.ablasser@epfl.ch 


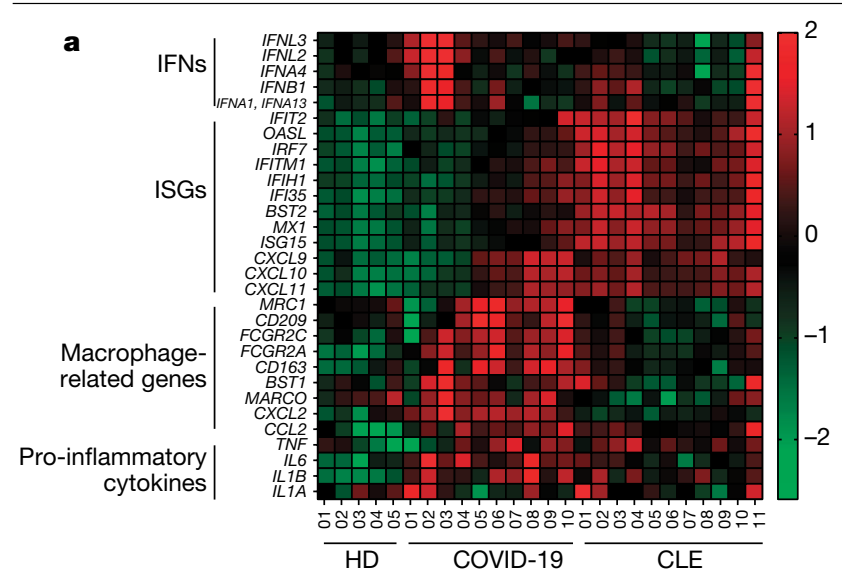

e
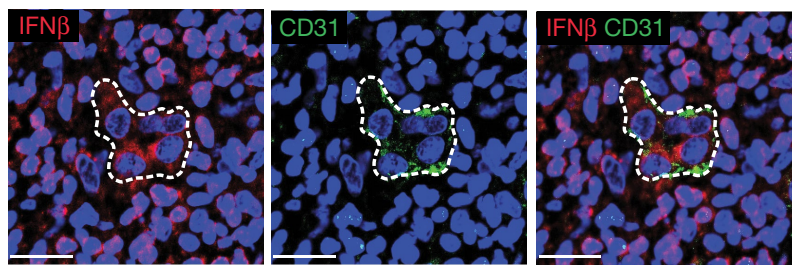

h
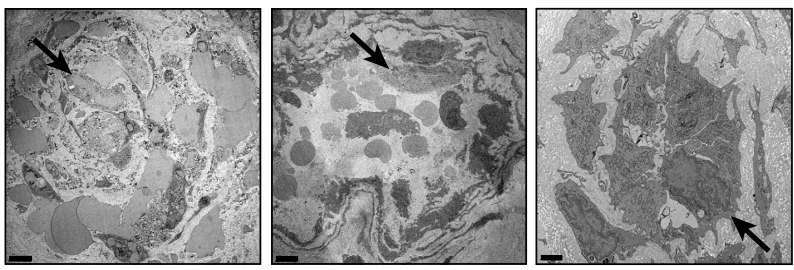

Fig. 1 | Type I IFN-producing macrophages surround damaged endothelial cells in COVID-19 skin lesions. a, Immune gene expression profiles of skin lesions from individuals with COVID-19 $(n=10)$ and individuals with CLE $(n=11)$, and skin from healthy donors (HD; $n=5)$. Unbiased clustering was performed. b, Immunohistochemistry quantification of macrophages, neutrophils, plasmacytoid dendritic cells (pDCs) and T cells (stained for CD163, MPO, CD123 and CD3) in CLE $(n=5)$ and COVID-19 $(n=10)$ skin lesions. c, Confocal microscopy images of representative COVID-19 skin lesion stained for CD163 (green) and IFN $\beta$ (red). Scale bars, $20 \mu \mathrm{m}$.d, Contribution of CD163 ${ }^{+}$ macrophages and CD $31^{+}$endothelial cells to IFN $\beta$ expression in CLE $(n=5)$ and COVID-19 $(n=10)$. e, Confocal microscopy images of representative COVID-19 skin lesion stained for CD31 (green) and IFN $\beta$ (red). Scale bars, $20 \mu \mathrm{m}$.

f, Proportions of $\mathrm{CD}_{163}{ }^{+}$macrophages, $\mathrm{CD} 31^{+}$endothelial cells and other cells among IFN $\beta$-producing cells for each CLE and COVID-19 sample.g, Confocal microscopy images of a representative COVID-19 skin sample stained for CD163
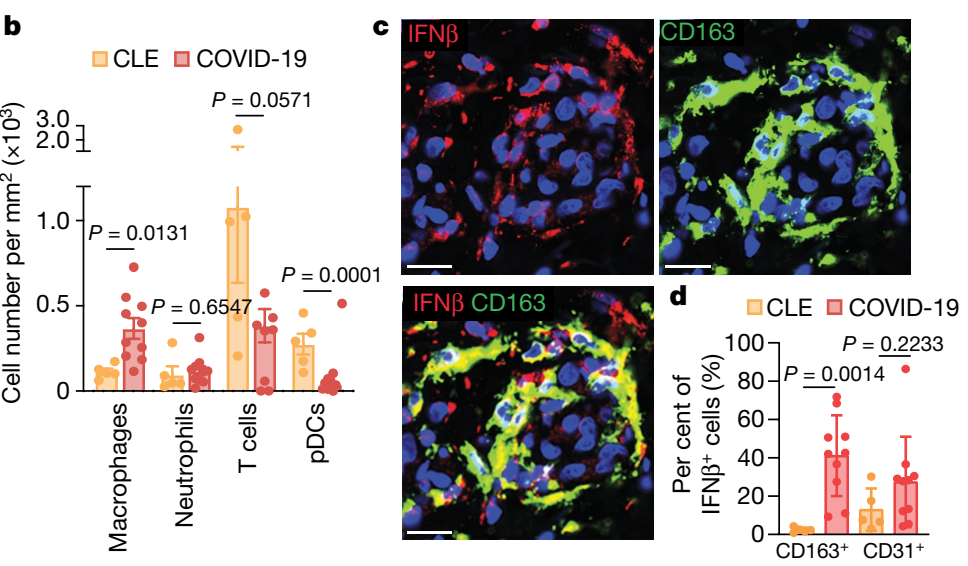

f $\square$ Other cells $\square$ Endothelial cells $\square$ Macrophages

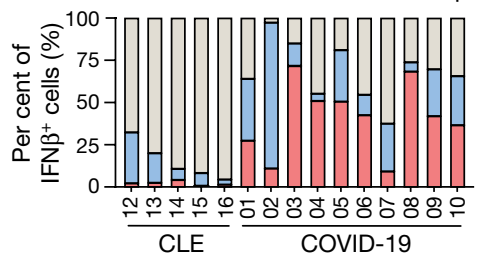

i
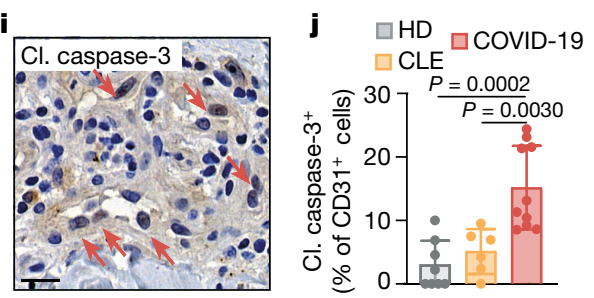
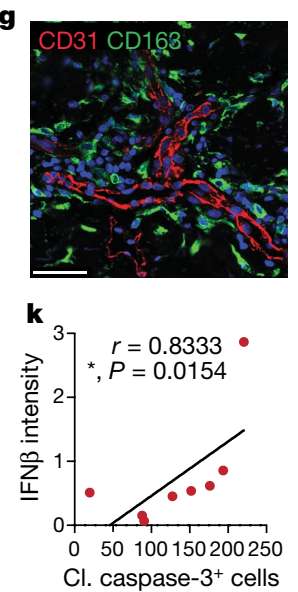

(green) and CD31 (red) to depict macrophages and endothelial cells. Scale bar, $50 \mu \mathrm{m} . \mathbf{h}$, Transmission electron microscopy of dermal vessels in purpuro-necrotic (left) and maculopapular (middle) COVID-19 skin lesions, and in healthy skin (right). Arrows show disrupted endothelial cells (COVID-19 skin lesions) and intact endothelial cells (healthy skin). Scale bars, $2 \mu \mathrm{m}$ (left); $5 \mu \mathrm{m}$ (middle); $1 \mu \mathrm{m}$ (right). i, Immunohistochemistry for cleaved caspase-3 (cl. caspase-3) in COVID-19 skin lesions (nuclear staining indicated by arrow). Scale bar, $20 \mu \mathrm{m}$.j, Percentage of CD31 ${ }^{+}$endothelial cells with cleaved caspase-3 staining in healthy skin $(n=8)$ and in CLE $(n=6)$ and COVID-19 $(n=10)$ skin lesions. k, Correlation between cleaved-caspase-3-positive nuclei and overall staining intensity of IFN $\beta$ measured in COVID-19 skin samples $(n=8)$. Spearman correlation and two-tailed statistical significance were performed. Data are mean \pm s.d. $(\mathbf{b}, \mathbf{d}, \mathbf{j})$. $P$ values obtained with two-tailed Student's $t$-test and one-way ANOVA followed by Tukey's multiple comparisons test $(\mathbf{b}, \mathbf{d}, \mathbf{j})$.

accumulated inside macrophages, especially in cells adjacent to the vasculature (Extended Data Fig. 4). In addition, we observed intracellular DNA foci accumulating inside IFN $\beta$-producing macrophages (Fig. 2a, b, Extended Data Fig. 4b). On the basis of these findings, we considered that engagement of the cGAS-STING pathway, a pivotal cytosolic DNA sensing mechanism, triggers the activation of macrophages ${ }^{18}$. After binding DNA, cGAS synthesizes the second messenger cyclic GMP-AMP (cGAMP), which activates STING to induce cytokine responses, including type IIFNs ${ }^{18}$. To directly address the involvement of cGAS, we measured levels of cGAMP in whole skin extracts. Samples from patients with COVID-19, but not those from healthy donors, showed increased levels of cGAMP (Fig. 2c). Consistent with the activation of cGAS-STING signalling, phosphorylated STING ( $p$-STING)-a selective marker of activated $\mathrm{STING}^{23}$ - was observed in perivascular macrophages in COVID-19 lesions, but not in healthy controls (Fig. 2d, e). In addition, STING was phosphorylated in endothelial cells (Fig. 2f, g), which also contribute to type IIFN production (see above). Finally, we 

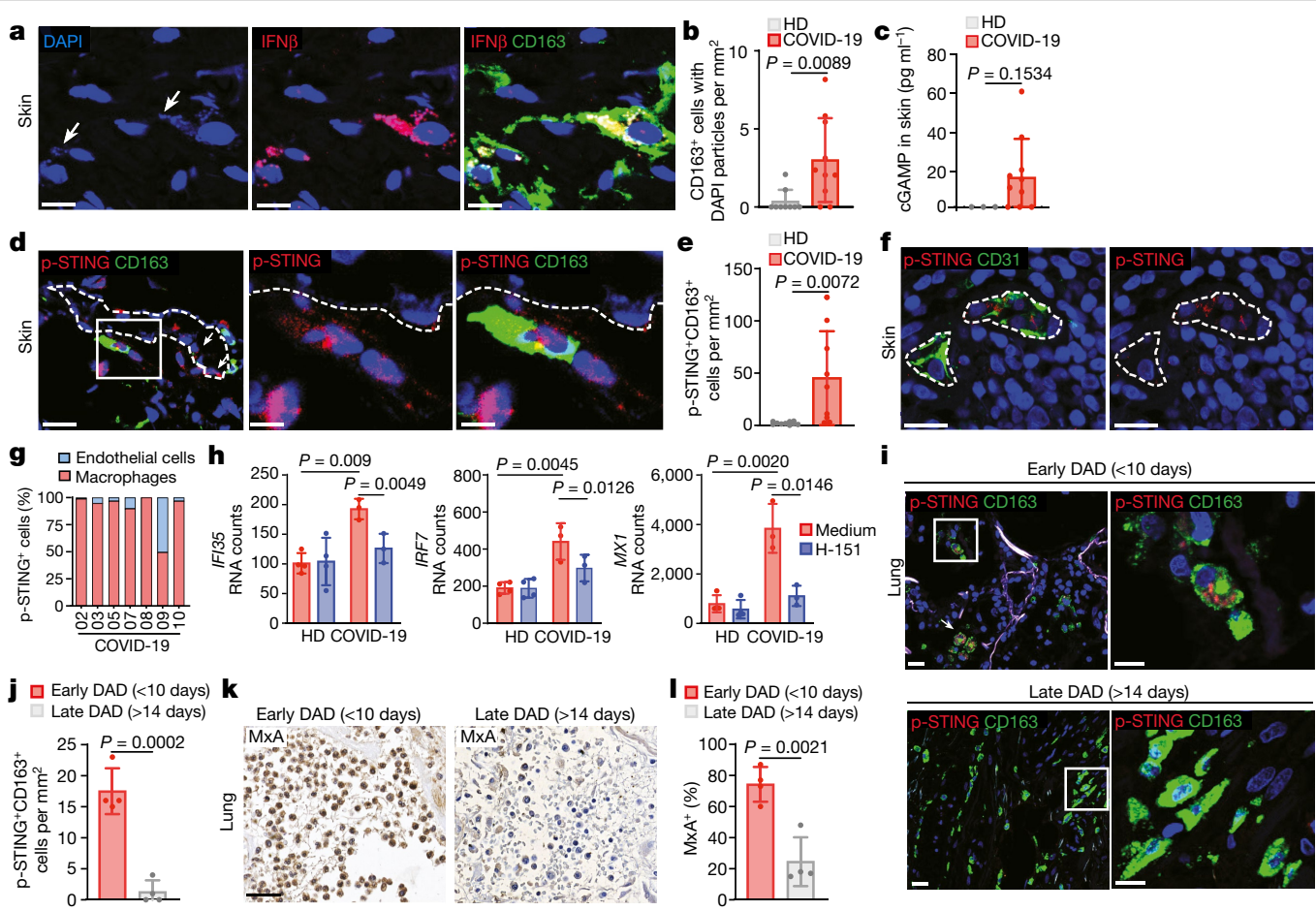

Fig. 2 | cGAS-STING-dependent type I IFN signature in COVID-19 skin and lung pathology. a, Confocal microscopy images of a representative COVID-19 skin sample stained for CD163 (green), IFNB1 mRNA (red) and DNA (blue). Scale bars, $10 \mu \mathrm{m}$. Arrows indicate cytosolic DNA particles. b, Quantification of $\mathrm{CD}_{163}{ }^{+}$macrophages containing cytosolic DNA particles in COVID-19 skin lesions $(n=10)$ and in healthy skin $(n=9)$. c, Quantification of cGAMP in lysates of COVID-19 skin lesions $(n=10)$ and healthy skin $(n=3)$. d, Confocal microscopy images of a representative COVID-19 skin sample stained for CD163 (green) and p-STING (red). Blood vessels, dashed line. Arrows show p-STING ${ }^{+}$endothelial cells. Scale bars, $20 \mu \mathrm{m}$ (left); $5 \mu \mathrm{m}$ (right two images). e, Quantification of p-STING ${ }^{+}$macrophages in COVID-19 skin lesions $(n=10)$ and in healthy skin $(n=10)$.f, Confocal microscopy images of a representative COVID-19 skin sample stained for CD31 (green) and p-STING (red). Blood vessels, dashed line.
Scale bars, $20 \mu \mathrm{m} . \mathrm{g}$, Proportions of $\mathrm{CD} 163^{+}$macrophages and CD $31^{+}$endothelial cells among $\mathrm{p}-\mathrm{STING}^{+}$cells in COVID-19 skin lesions $(n=7)$. h, Expression of ISGs (IFI35, IRF7 and MX1) in cultured healthy skin $(n=3)$ and COVID-19 skin explants $(n=3)$, treated or not with $\mathrm{H}-151 . \mathbf{i}$, Confocal microscopy images of representative post-mortem lungs with early (fewer than 10 days; left) or late (more than 14 days, right) DAD, stained for p-STING (red) and CD163 (green). Scale bars (left to right): $20 \mu \mathrm{m}, 10 \mu \mathrm{m}, 50 \mu \mathrm{m}, 10 \mu \mathrm{m}$.j, Quantification of p-STING ${ }^{+} \mathrm{CD}_{163}{ }^{+}$macrophages in post-mortem lungs with early and late DAD $(n=4) . \mathbf{k}$, Immunohistochemistry of representative post-mortem lungs with early (left) or late (right) DAD, stained for MxA. Scale bar, $50 \mu \mathrm{m}$. I, Percentage of tissue area with MxA positivity in early and late DAD samples $(n=4)$. Data are mean \pm s.d. $(\mathbf{b}, \mathbf{c}, \mathbf{e}, \mathbf{h}, \mathbf{j}, \mathbf{I})$. $P$ values obtained with two-tailed Student's t-test $(\mathbf{c}, \mathbf{e}, \mathbf{h}, \mathbf{j}, \mathbf{l})$ and with Mann-Whitney test (b). cultured COVID-19 skin explants overnight in the presence or absence of a small-molecule STING inhibitor, $\mathrm{H}-151$ ( ref. $^{24}$ ). Compared to healthy skin, COVID-19 explants exhibited significant expression of ISGs (IFI35, IRF7 and $M X 1$ ), and this response was strongly reduced by $\mathrm{H}-151$ (Fig. $2 \mathrm{~h}$ ). Thus, the cGAS-STING pathway is a crucial driver of type IIFN responses in COVID-19 skin lesions.

We next sought to determine whether cGAS-STING activation also occurs in severely damaged lungs of patients with COVID-19 by post-mortem analysis ${ }^{25}$ (Extended Data Fig. 5a,b). We observed p-STING in macrophages and endothelial cells in some-but not all-of the lung autopsies that were analysed (Fig. 2i, j, Extended Data Fig. 5c, d). Further histopathological examination showed that lung samples that exhibited p-STING expression belonged to patients with a rapidly lethal disease course (death at fewer than 10 days after disease onset), and were characterized by signs of early diffuse alveolar damage (DAD) with extensive hyaline membrane formation (Fig. 2i, j, Extended Data Fig. 5a,b). By contrast, samples that lacked p-STING were from patients with a protracted disease course (death at more than 14 days after disease onset) and exhibited fibrotic changes that are characteristic of later phases of $\operatorname{DAD}^{25}$ (Fig. 2i, j, Extended Data Fig. 5a, b). In addition, samples with hallmarks for early DAD, but not late DAD, showed a type I IFN signature, as indicated by increased expression of MxA (Fig. $2 \mathrm{k}, \mathrm{l}$ ). Together, these analyses link SARS-CoV-2-induced tissue damage in the lung to the activation of the cGAS-STING pathway and type IIFN signalling.

\section{Endothelial STING response to infection}

The above results suggested that, besides macrophages, endothelial cells might contribute to STING-dependent type I IFN responses in COVID-19. Although SARS-CoV-2 affects the vascular endothelium in patients, poor in vitro infection of endothelial cell cultures prevents the study of infection-associated processes in these cells ${ }^{26-28}$. To overcome this limitation and determine the role of STING in the endothelium, we used a lung-on-chip (LoC) model, which mimics the alveolar-capillary interface and allows for robust SARS-CoV-2-dependent activities in endothelial cells ${ }^{27}$ (Fig. 3a, Extended Data Fig. 6a). After infection of the alveolar epithelium, endothelial cells-but not epithelial cells-produced high levels of IFN $\beta$, and this response was completely abolished when the STING inhibitor $\mathrm{H}-151$ was perfused through the vascular channel (Fig. 3b, c, Extended Data Fig. 6b, c). Consistent with a direct engagement of STING, endothelial cells contained perinuclear foci of $p$-STING after infection (Fig. 3d). In addition, we verified that macrophages could contribute to the resultant type IIFN response on the vascular side in a manner that was dependent on cGAS (Fig. 3b, Extended Data Fig. 6d). Of note, we observed that the prominent virus-induced cytopathic effect in endothelial cells was also sensitive to treatment with $\mathrm{H}-151$ (Fig. 3c, Extended Data Fig. 6e). By short hairpin RNA (shRNA)-mediated knockdown, we confirmed that commitment to infection-induced cell death depended on STING in endothelial cells, whereas depleting STING in the epithelial layer did not affect cell viability (Extended 


\section{Article}

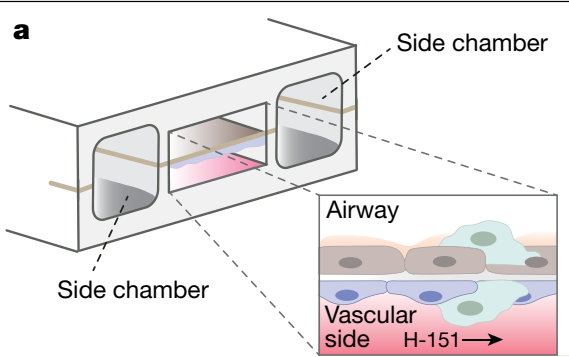

c SARS-CoV-2
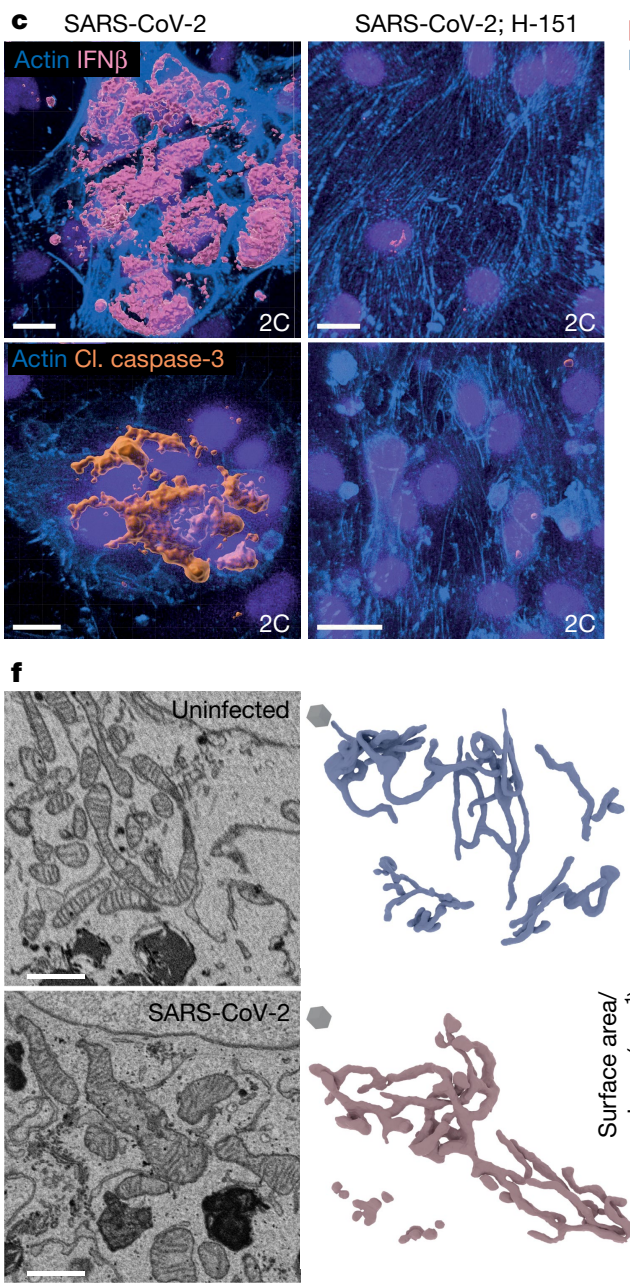

Uninfected

$\square$ SARS-CoV-2
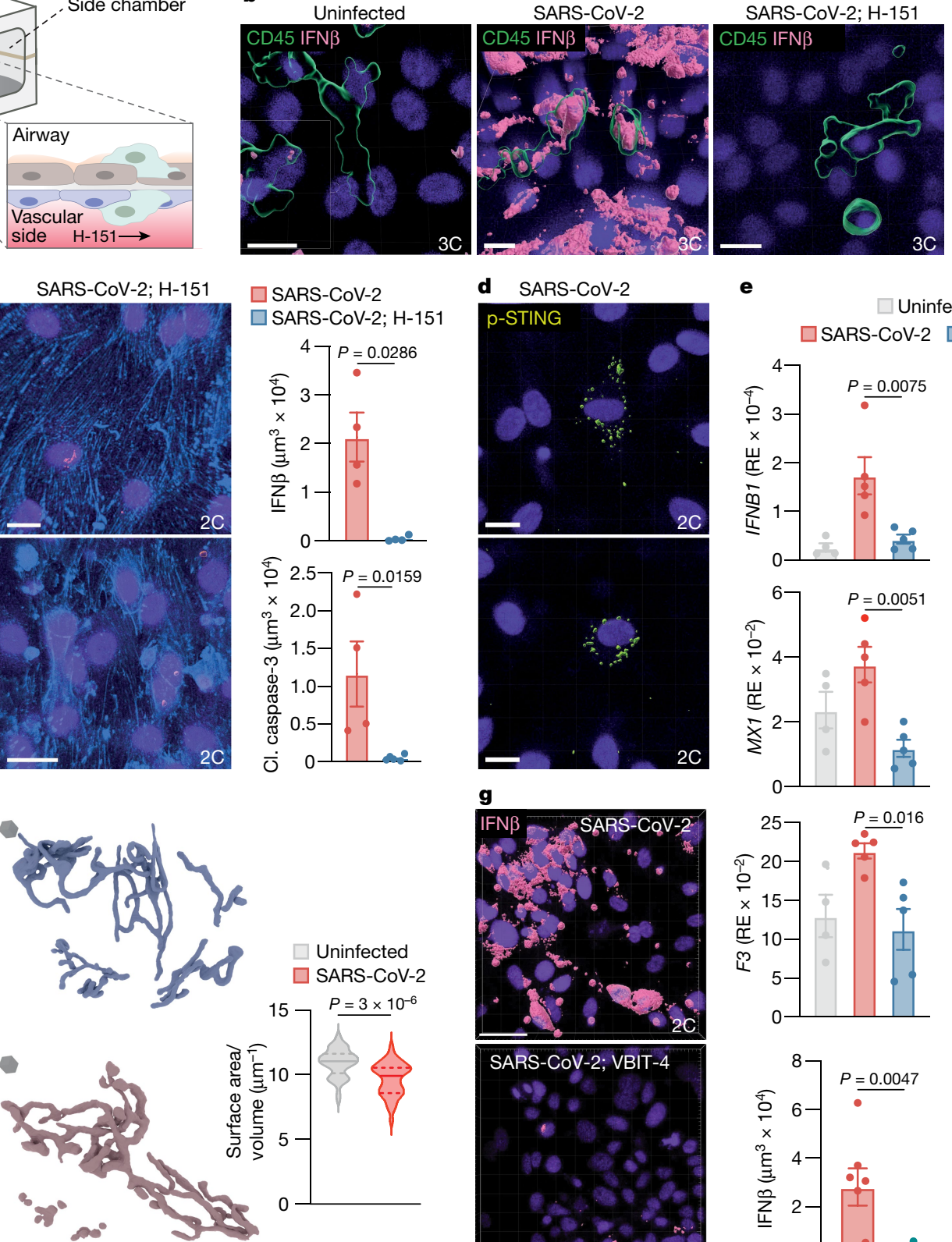

d SARS-CoV-2 e

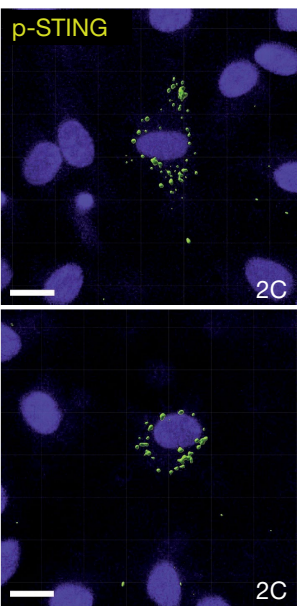

e

$3 \mathrm{C}$

$\square$ Uninfected

$\square$ SARS-CoV-2 $\square$ SARS-CoV-2; H-151
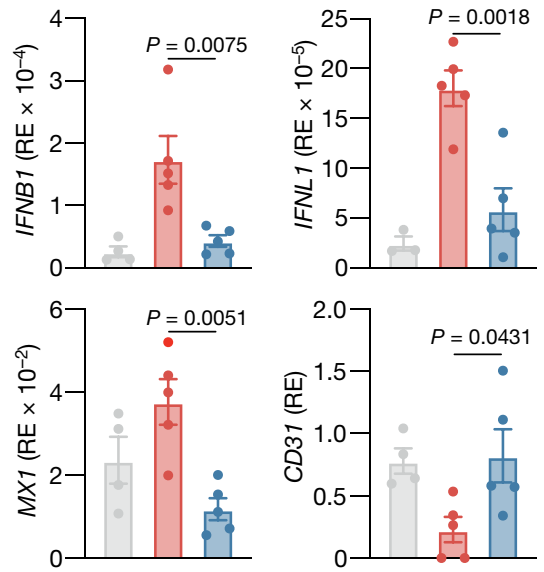

g
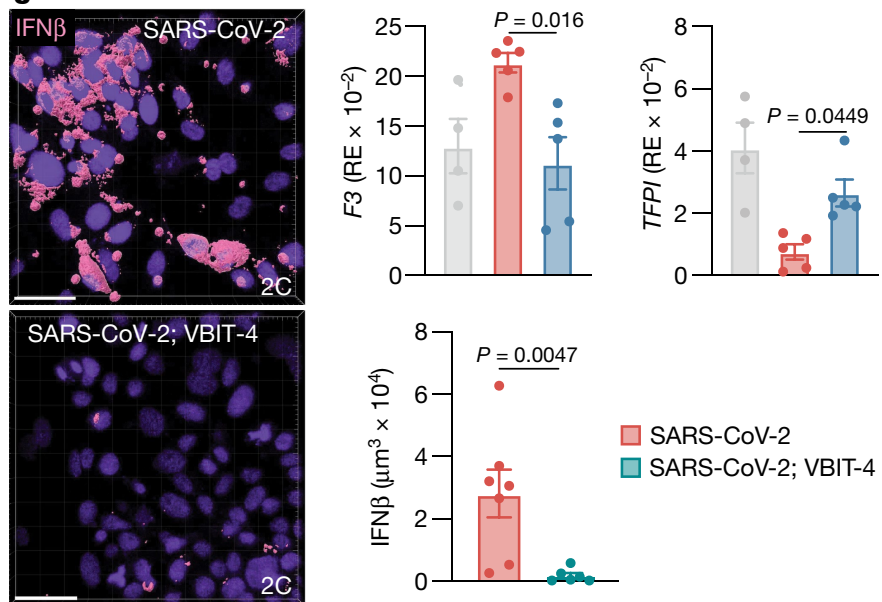

$\square$ SARS-CoV-2

$\square$ SARS-CoV-2; VBIT-4
Fig. 3 | STING-dependent type I IFN production and cell death after SARS-CoV-2 infection in endothelial cells. a, Schematic of the three-cell component LoC model.b, c, Representative 3D images of the vascular face of uninfected or SARS-CoV-2-infected LoCs with or without vascular H-151 perfusion. '3C', three-cell component (epithelial cells, endothelial cells and macrophages) in b; ' $2 C^{\prime}$ ', two-cell component (epithelial cells and endothelial cells) in c. $\mathrm{CD}_{4} 5^{+}$macrophage (green), IFN $\beta$ (bright pink), cleaved caspase-3 (amber), actin (azure) and nuclear (purple) stainings are shown. Scale bars, $20 \mu \mathrm{m}$. d, Representative 3D images of p-STING ${ }^{+}$endothelial cells (yellow). Scale bars, $20 \mu \mathrm{m}$. e, Expression levels of the indicated genes in uninfected $(n=4)$, infected $(n=5)$ and H-151-treated $(n=5)$ LoCs. CD31 is also known as PECAM1. RE, relative expression.f, Representative volumetric electron microscopy images, 3D reconstructions and quantification of the

Data Fig. 7a). Further transcriptional analysis revealed changes of endothelial-specific activation markers (F3, TFPI and CD31), which were regulated by STING as $\mathrm{H}-151$ effectively suppressed this response (Fig. 3e). As a control, treatment with $\mathrm{H}-151$ did not affect SARS-CoV-2 transcript expression in endothelial cells (Extended Data Fig. 6a). We also tested the involvement of RNA-sensing RIG-I-like receptors surface-area-to-volume ratio of endothelial cell mitochondria from uninfected $(n=45)$ and infected $(n=43)$ LoCs. Solid line, mean; dashed lines, quartiles. Scale bars, $1 \mu \mathrm{m}$ (left images); grey hexagons (middle images) represent $1 \mu \mathrm{m}^{3}$. $\mathbf{g}$, Representative 3D images of the vascular face of infected LoCs with or without vascular VBIT-4 perfusion. Scale bars, $50 \mu \mathrm{m}$. Statistics for quantification: b, IFN $\beta$ : uninfected ( $n=6$ fields of view (FOV)), infected $(n=7$ FOV) and H-151-treated ( $n=4$ FOV) LoCs, $n=2$ LoCs each; c, IFN $\beta /$ cleaved caspase- 3 : infected ( $n=4 \mathrm{FOV}$ in each case) and H-151-treated ( $n=4 / n=5 \mathrm{FOV}$ ) LoCs, $n=2$ LoCs each for both markers; f, data from $n=4$ endothelial cells each from $n=1$ uninfected and $n=2$ infected LoCs; g, IFN $\beta$ : infected $(n=7$ FOV) and VBIT-4-treated $(n=6 \mathrm{FOV})$ LoCs, $n=2$ LoCs each. Data acquired at $3 \mathrm{dpi}$; mean \pm s.e.m.; $P$ values calculated by one-way ANOVA followed by Tukey's multiple comparisons tests (b, c, e) or two-tailed Mann-Whitney test (f, $\mathbf{g})$.

and found that a knockdown of mitochondrial antiviral signalling protein (MAVS) in endothelial cells left the type I IFN response unaffected (Extended Data Fig. 7b). Together, these data reveal that STING participates in the response of endothelial cells towards SARS-CoV-2 infection by controlling distinct effector programs; namely, type IIFN signalling and endothelial cell death. 

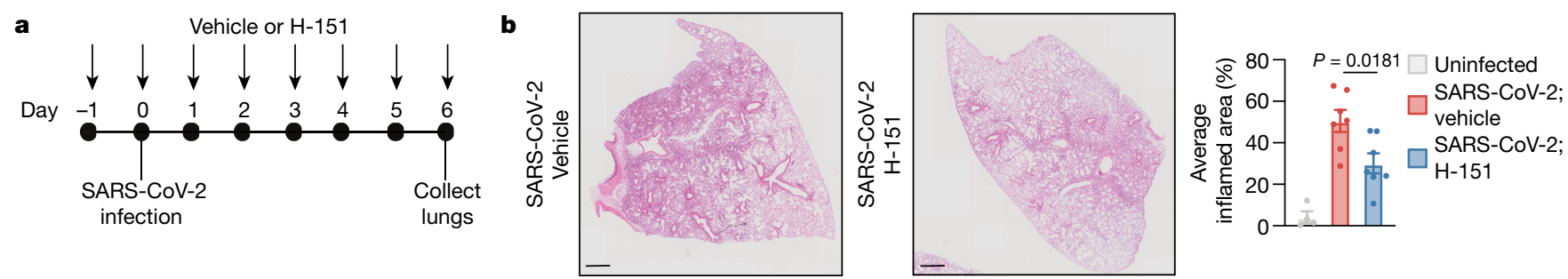

c ISGs
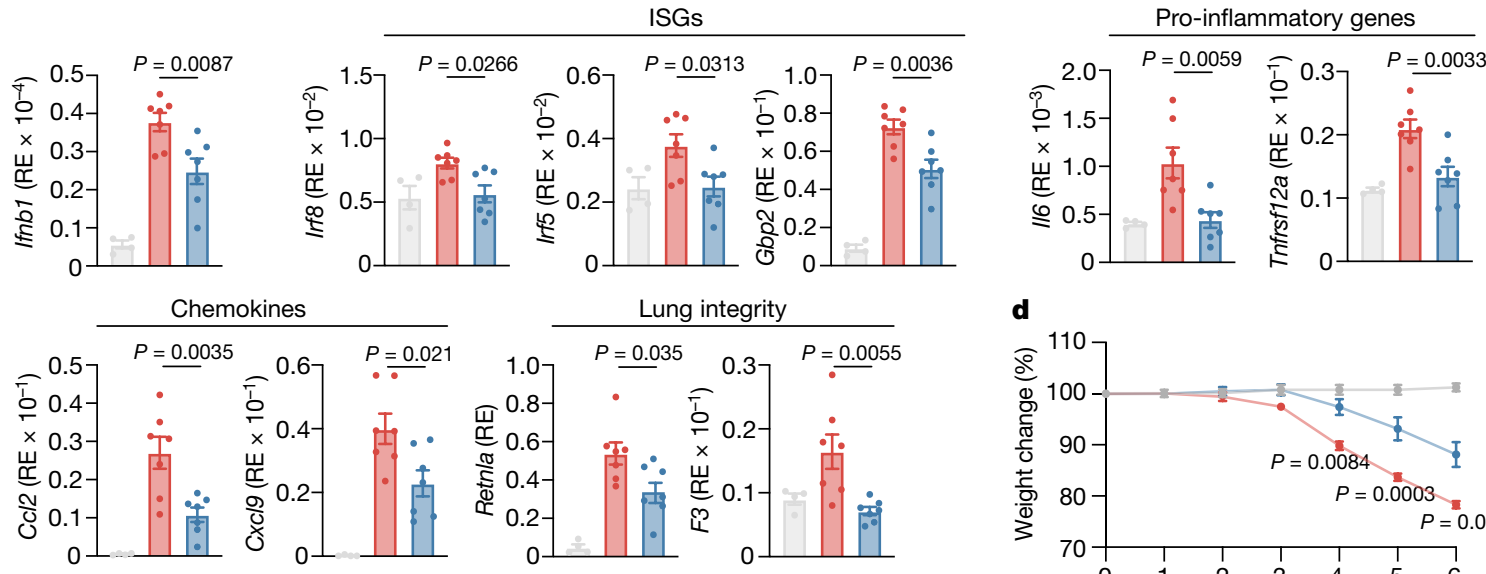

d
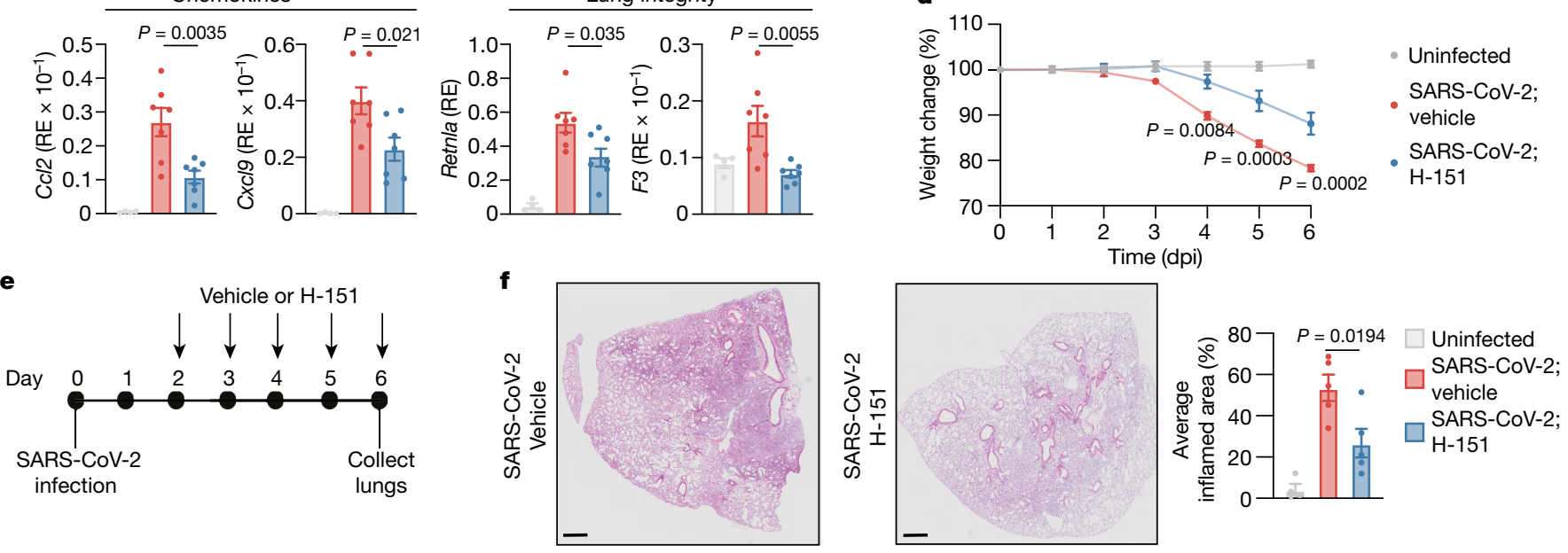

g

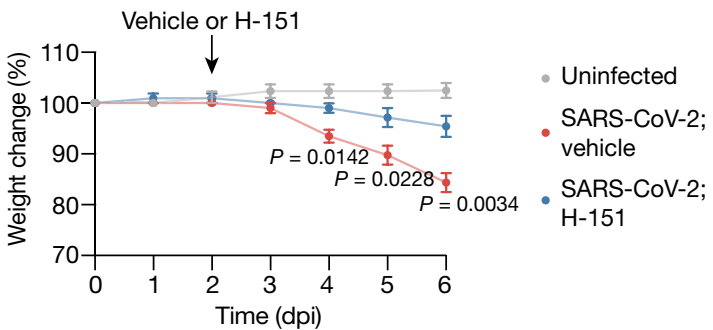

Fig. 4 | STING inhibition reduces SARS-CoV-2-induced inflammation in mice. a, Schematic of SARS-CoV-2 infection (intranasal; $1 \times 10^{4}$ plaque-forming units (PFU) per mouse) and intraperitoneal administration of vehicle or $\mathrm{H}-151$ (starting at 1 day before infection), related to data from $\mathbf{b}-\mathbf{d} . \mathbf{b}$, Left, representative haematoxylin and eosin (H\&E) images of lungs from vehicle- and H-151-treated mice. Scale bars, $500 \mu \mathrm{m}$. Right, average inflamed area in SARS-CoV- 2 infected mice.c, mRNA expression levels of the indicated genes in uninfected and infected lungs at $6 \mathrm{dpi}$, analysed by RT-qPCR.d, Relative weight loss in mice after SARS-CoV-2 infection. e, Schematic of SARS-CoV- 2 infection (intranasal; $1 \times 10^{4}$ PFU per mouse) and intraperitoneal administration of vehicle or H-151 (starting

We next investigated the mechanism that governs STING activation in endothelial cells. To this end, we profiled the cytosol of endothelial cells after LoC infection for changes in highly expressed proteins by mass spectrometry. Analysis of the mass spectrometry data identified differences in the abundance of 75 proteins at 3 days after infection, with a particular enrichment of mitochondrial proteins (that is, mitochondrial proteins linked to the Gene Ontology (GO) terms 'thermogenesis' or 'oxidative phosphorylation') (Extended Data Fig. 8a, Supplementary Table 3). Time-course analysis confirmed a steady increase in altered expression of proteins linked to mitochondrial metabolism (Extended
Data Fig. 8b). Moreover, in volumetric ultrastructural imaging in situ, the mitochondria of endothelial cells showed disrupted cristae and appeared swollen after infection, with a pronounced reduction in the surface-area-to-volume ratio (Fig. 3f, Extended Data Fig. 8c). Notably, endothelial cells containing damaged mitochondria were also detected in skin biopsies from patients with COVID-19 (Extended Data Fig. 8d). On the basis of these findings, we hypothesized that mitochondrial DNA (mtDNA) released into the cytosol might trigger cGAS upstream of STING in endothelial cells. To test this idea, we incubated endothelial cells with $2^{\prime}, 3^{\prime}$-dideoxycytidine (ddC) to deplete mtDNA ( $\rho^{0}$ cells) 
(Extended Data Fig. 8e). Compared to control cells, $\rho^{0}$ cells showed significantly less production of type I IFNs after epithelial infection with SARS-CoV-2 (Extended Data Fig. 8d). In addition, inhibition of VDAC1 oligomerization by VBIT-4, which enables the passage of mtDNA fragments into the cytosol during mitochondrial stress ${ }^{29}$, decreased the production of type I IFNs in endothelial cells (Fig. 3g). Therefore, extending previous findings ${ }^{30,31}$, SARS-CoV-2 can provoke mitochondrial dysfunction, which in endothelial cells connects to activation of the cGAS-STING pathway through the release of endogenous mtDNA.

\section{Targeting STING in a mouse model of COVID-19}

To investigate the role of STING during SARS-CoV-2 infection in vivo, we used K18-hACE2 transgenic mice, which are highly susceptible to SARS-CoV-2 infection and recapitulate important immunological features of severe COVID-19 in humans ${ }^{32-36}$. K18-hACE2 mice received one daily dose of $\mathrm{H}-151$ starting at $16 \mathrm{~h}$ before SARS-CoV-2 infection and were euthanized at 3 days or 6 days post infection (dpi) (Fig. 4a). Histological examination of the lungs showed a significant reduction of inflammatory cell infiltration in $\mathrm{H}$-151-treated compared to vehicle-treated mice at $6 \mathrm{dpi}$ (Fig. 4b). As an independent measure of tissue pathology, we observed a prominent accumulation of dying cells (Extended Data Fig. 9a). Cell death was efficiently blocked by treatment with $\mathrm{H}-151$ at $6 \mathrm{dpi}$, but not at $3 \mathrm{dpi}$ (Extended Data Fig. 9a). These findings show that STING is a critical contributor to SARS-CoV-2-induced lung pathology.

When examining cytokine responses in the lungs after infection, we found that treatment with $\mathrm{H}-151$ considerably decreased the expression of Ifnb1 and ISGs (for example, Gbp2, Irf5 and Irf8) at $6 \mathrm{dpi}^{32,35}$ (Fig. 4c, Extended Data Fig. 9b). Levels of pro-inflammatory genes (Il6 and Tnfrsf12a), chemokines ( $\mathrm{Ccl}, \mathrm{Ccl} 3, \mathrm{Ccl12}$ and $\mathrm{Cxcl9})$ and markers of lung injury ( $F 3$ and Retnla) were also significantly lower in $\mathrm{H}$-151-treated compared to vehicle-treated mice ${ }^{35}$ (Fig. 4c, Extended Data Fig. 9b). Furthermore, lung homogenates collected at $6 \mathrm{dpi}$ showed strongly reduced activity of NF- $\mathrm{KB}$ and type I IFN signalling, as shown by decreased levels of p-p65 and p-STAT1, respectively (Extended Data Fig. 9c). Viral replication was similar in the presence or absence of $\mathrm{H}-151$ at each time point, ruling out a significant effect of STING inhibition on viral replication (Extended Data Fig. 9d). Notably, at 3 dpi there was no appreciable difference in cytokine levels in the lungs between the two treatment groups (Extended Data Fig. 9b). Collectively, these data show that STING has a unique and critical function in eliciting type IIFN and inflammatory responses in the late phase of infection, which coincides with excessive tissue damage, but not with the peak of viral replication ${ }^{32}$. We also monitored body weight changes of infected mice over time. Compared to treatment with vehicle, treatment with $\mathrm{H}-151$ significantly attenuated weight loss after infection, showing that STING has an essential role in the progression to severe disease (Fig. 4d).

Finally, we determined the effect of $\mathrm{H}-151$ as a therapeutic agent in ongoing disease when viral loads are maximal ${ }^{32}$ (Fig. $4 \mathrm{e}$ ). Mice that received $\mathrm{H}-151$ at $2 \mathrm{dpi}$ showed reduced pathology and decreased levels of type IIFNs and other cytokines in the lungs compared to those that were treated with vehicle only, whereas viral loads were similar between the two groups (Fig. 4f, Extended Data Fig. 10a, b). Therapeutic administration of $\mathrm{H}-151$ also protected mice from weight loss and death after infection with SARS-CoV-2 (Fig. 4g, h). Together with the data above, these results corroborate the select role of STING in promoting detrimental inflammation during the late (or later) stages of the infection and highlight the therapeutic efficacy of STING inhibition, whether in a prophylactic or a therapeutic setting.

\section{Discussion}

We have identified a central mechanism of innate immunopathology in COVID-19. Our study shows that engagement of the cGAS-STING pathway regulates two distinctive pathological features that are critically involved in the progression and severity of COVID-19-namely, endothelial dysfunction and type I IFN production (Extended Data Fig. 10c). Moreover, we establish endothelial cells and macrophages as cells at the root of maladapted cGAS-STING responses, which are driven in each cell type by a distinct underlying process. In a cell-intrinsic mode of activation, cGAS within endothelial cells is stimulated by the loss of mitochondrial homeostasis and associated accumulation of mtDNA to direct the expression of type IIFNs, activation of endothelial cells and, ultimately, cell death. By contrast, macrophage-dependent cGAS responses are more focused on type I IFN induction and result from the recognition of DNA from engulfed dying (endothelial) cells. Our finding that STING regulates endothelial cell death accords well with previous reports of endotheliopathy and vascular damage due to gain-of-function mutations in STING or after administration of highly potent STING agonists ${ }^{37,38}$. Consistent with previous reports in patients with COVID-19 (ref. ${ }^{26}$ ), endothelial cells in our LoC studies contain viral elements, arguing for direct viral involvement in triggering mitochondrial dysfunction and, in turn, the activity of the cGAS-STING pathway. Along these lines, cell-autonomous activation of cGAS-STING signalling has recently been suggested to contribute to the NF-kB-dependent production of cytokines in SARS-CoV-2-infected human epithelial cell lines ${ }^{39}$, which indicates that the pathway could have a more extensive role in COVID-19-associated cytokine responses. Given the importance of vascular damage in COVID-19, establishing the precise upstream cause (or causes) of endothelial cell involvement in viral activities is an area that warrants future investigation ${ }^{21,40}$.

Many reports have pointed to the context-dependent roles of type I IFNs during infection with highly pathogenic coronaviruse ${ }^{3,5,6,9-11,17,41,42}$. Our study adds to these reports by indicating that the signalling mechanisms that underlie the induction of beneficial (early) versus detrimental (delayed) type I IFN responses are distinct. In a direct mode of recognition, rapid detection of viral RNA by Toll-like receptors 3 and 7 and RIG-I-like receptors initiates a type I IFN response that confers antiviral protection to the host ${ }^{43-46,47}$. By contrast, activation of the cGAS-STING pathway by DNA emerges from a collateral host response to tissue damage. This explains the involvement of the pathway in type I IFN production at the later stages of infection, eventually sustaining deleterious inflammation. Notably, STING-dependent induction of type I IFNs is compromised in bats, which raises the possibility that this immunological adaption could account for the increased tolerance of these animals to highly pathogenic coronavirus infection ${ }^{48}$.

In summary, our study has implications both for the understanding of how the innate immune system contributes to detrimental outcomes of SARS-CoV-2 infection and for current efforts to define new therapeutic paradigms for more efficient treatment modalities in COVID-19.

\section{Online content}

Any methods, additional references, Nature Research reporting summaries, source data, extended data, supplementary information, acknowledgements, peer review information; details of author contributions and competing interests; and statements of data and code availability are available at https://doi.org/10.1038/s41586-022-04421-w.

1. Huang, C. et al. Clinical features of patients infected with 2019 novel coronavirus in Wuhan, China. Lancet 395, 497-506 (2020).

2. Gupta, A. et al. Extrapulmonary manifestations of COVID-19. Nat. Med. 26, 1017-1032 (2020).

3. Lucas, C. et al. Longitudinal analyses reveal immunological misfiring in severe COVID-19. Nature 584, 463-469 (2020).

4. Nienhold, R. et al. Two distinct immunopathological profiles in autopsy lungs of COVID-19. Nat. Commun. 11, 5086 (2020).

5. Park, A. \& Iwasaki, A. Type I and type III interferons-induction, signaling, evasion, and application to combat COVID-19. Cell Host Microbe 27, 870-878 (2020).

6. Lee, J. S. \& Shin, E.-C. The type I interferon response in COVID-19: implications for treatment. Nat. Rev. Immunol. 20, 585-586 (2020).

7. Hadjadj, J. et al. Impaired type I interferon activity and inflammatory responses in severe COVID-19 patients. Science 369, 718-724 (2020). 
8. Chua, R. L. et al. COVID-19 severity correlates with airway epithelium-immune cell interactions identified by single-cell analysis. Nat. Biotechnol. 38, 970-979 (2020).

9. Zhou, Z. et al. Heightened innate immune responses in the respiratory tract of COVID-19 patients. Cell Host Microbe 27, 883-890 (2020).

10. Wilk, A. J. et al. A single-cell atlas of the peripheral immune response in patients with severe COVID-19. Nat. Med. 26, 1070-1076 (2020).

11. Lee, J. S. et al. Immunophenotyping of COVID-19 and influenza highlights the role of type I interferons in development of severe COVID-19. Sci. Immunol. 5, eabd1554 (2020).

12. Schulte-Schrepping, J. et al. Severe COVID-19 is marked by a dysregulated myeloid cell compartment. Cell 182, 1419-1440 (2020).

13. Galani, I.-E. et al. Untuned antiviral immunity in COVID-19 revealed by temporal type I/III interferon patterns and flu comparison. Nat. Immunol. 22, 32-40 (2021).

14. Wolfel, R. et al. Virological assessment of hospitalized patients with COVID-2019. Nature 581, 465-469 (2020).

15. Wang, N. et al. Retrospective multicenter cohort study shows early interferon therapy is associated with favorable clinical responses in COVID-19 patients. Cell Host Microbe 28, 455-464 (2020).

16. Israelow, B. et al. Mouse model of SARS-CoV-2 reveals inflammatory role of type I interferon signaling. J. Exp. Med. 217, e20201241 (2020).

17. Liao, M. et al. Single-cell landscape of bronchoalveolar immune cells in patients with COVID-19. Nat. Med. 26, 842-844 (2020).

18. Ablasser, A. \& Chen, Z. J. cGAS in action: expanding roles in immunity and inflammation. Science 363, eaat8657 (2019).

19. Farber, D. L. Tissues, not blood, are where immune cells function. Nature 593, 506-509 (2021).

20. Freeman, E. E. et al. The spectrum of COVID-19-associated dermatologic manifestations: an international registry of 716 patients from 31 countries. J. Am. Acad. Dermatol. 83, $1118-1129$ (2020).

21. Goshua, G. et al. Endotheliopathy in COVID-19-associated coagulopathy: evidence from a single-centre, cross-sectional study. Lancet Haematol. 7, e575-e582 (2020).

22. Bonaventura, A. et al. Endothelial dysfunction and immunothrombosis as key pathogenic mechanisms in COVID-19. Nat. Rev. Immunol. 21, 319-329 (2021).

23. Liu, S. et al. Phosphorylation of innate immune adaptor proteins MAVS, STING, and TRIF induces IRF3 activation. Science 347, aaa2630 (2015).

24. Haag, S. M. et al. Targeting STING with covalent small-molecule inhibitors. Nature $\mathbf{5 5 9}$ 269-273 (2018).

25. Berezowska, S. et al. Postmortem cardiopulmonary pathology in patients with COVID-19 infection: single-center report of 12 autopsies from Lausanne, Switzerland. Diagnostics 11, 1357 (2021).

26. Varga, Z. et al. Endothelial cell infection and endotheliitis in COVID-19. Lancet 395, 1417-1418 (2020).

27. Thacker, V. V. et al. Rapid endotheliitis and vascular damage characterize SARS-CoV-2 infection in a human lung-on-chip model. EMBO Rep. 22, e52744 (2021).

28. Nascimento Conde, J., Schutt, W. R., Gorbunova, E. E. \& Mackow, E. R. Recombinant ACE2 expression is required for SARS-CoV-2 to infect primary human endothelial cells and induce inflammatory and procoagulative responses. mBio 11, e03185-20 (2020).

29. Kim, J. et al. VDAC oligomers form mitochondrial pores to release mtDNA fragments and promote lupus-like disease. Science 366, 1531-1536 (2019).

30. Stukalov, A. et al. Multilevel proteomics reveals host perturbations by SARS-CoV-2 and SARS-CoV. Nature 594, 246-252 (2021).

31. Gibellini, L. et al. Altered bioenergetics and mitochondrial dysfunction of monocytes in patients with COVID-19 pneumonia. EMBO Mol. Med. 12, e13001 (2020).
32. Winkler, E. S. et al. SARS-CoV-2 infection of human ACE2-transgenic mice causes severe lung inflammation and impaired function. Nat. Immunol. 21, 1327-1335 (2020).

33. McCray, P. B. Jr. et al. Lethal infection of K18-hACE2 mice infected with severe acute respiratory syndrome coronavirus. J. Virol. 81, 813-821 (2007).

34. Bao, L. et al. The pathogenicity of SARS-CoV-2 in hACE2 transgenic mice. Nature 583, 830-833 (2020).

35. Golden, J. W. et al. Human angiotensin-converting enzyme 2 transgenic mice infected with SARS-CoV-2 develop severe and fatal respiratory disease. JCI Insight 5, e142032 (2020).

36. Muñoz-Fontela, C. et al. Animal models for COVID-19. Nature 586, 509-515 (2020).

37. Liu, Y. et al. Activated STING in a vascular and pulmonary syndrome. N. Engl. J. Med. 371, 507-518 (2014).

38. Prantner, D. et al. 5,6-Dimethylxanthenone-4-acetic acid (DMXAA) activates stimulator of interferon gene (STING)-dependent innate immune pathways and is regulated by mitochondrial membrane potential. J. Biol. Chem. 287, 39776-39788 (2012).

39. Neufeldt, C. J. et al. SARS-CoV-2 infection induces a pro-inflammatory cytokine response through cGAS-STING and NF-KB. Commun. Biol. 5, 45 (2022).

40. Teuwen, L.-A., Geldhof, V., Pasut, A. \& Carmeliet, P. COVID-19: the vasculature unleashed. Nat. Rev. Immunol. 20, 389-391 (2020).

41. Channappanavar, R. et al. Dysregulated type I interferon and inflammatory monocytemacrophage responses cause lethal pneumonia in SARS-CoV-infected mice. Cell Host Microbe 19, 181-193 (2016).

42. Cameron, M. J. et al. Interferon-mediated immunopathological events are associated with atypical innate and adaptive immune responses in patients with severe acute respiratory syndrome. J. Virol. 81, 8692-8706 (2007).

43. Zhang, Q. et al. Inborn errors of type I IFN immunity in patients with life-threatening COVID-19. Science 370, eabd4570 (2020).

44. Yang, D. M., Geng, T. T., Harrison, A. G. \& Wang, P.-H. Differential roles of RIG-I-like receptors in SARS-CoV-2 infection. Military Med. Res. 8, 49 (2021).

45. Mazaleuskaya, L., Veltrop, R., Ikpeze, N., Martin-Garcia, J. \& Navas-Martin, S. Protective role of Toll-like receptor 3 -induced type I interferon in murine coronavirus infection of macrophages. Viruses 4, 901-923 (2012).

46. van der Made, C. I. et al. Presence of genetic variants among young men with severe COVID-19. JAMA 324, 663-673 (2020).

47. Asano, T. et al. X-linked recessive TLR7 deficiency in $\sim 1 \%$ of men under 60 years old with life-threatening COVID-19. Sci. Immunol. 6, eabl4348 (2021).

48. Xie, J. et al. Dampened STING-dependent interferon activation in bats. Cell Host Microbe 23, 297-301 (2018).

Publisher's note Springer Nature remains neutral with regard to jurisdictional claims in published maps and institutional affiliations.

(C) Open Access This article is licensed under a Creative Commons Attribution 4.0 International License, which permits use, sharing, adaptation, distribution and reproduction in any medium or format, as long as you give appropriate credit to the original author(s) and the source, provide a link to the Creative Commons license, and indicate if changes were made. The images or other third party material in this article are included in the article's Creative Commons license, unless indicated otherwise in a credit line to the material. If material is not included in the article's Creative Commons license and your intended use is not permitted by statutory regulation or exceeds the permitted use, you will need to obtain permission directly from the copyright holder. To view a copy of this license, visit http://creativecommons.org/licenses/by/4.0/.

(c) The Author(s) 2022 


\section{Methods}

\section{Patient data and samples}

Studies were approved by the institutional review and privacy boards of the Lausanne University Hospital CHUV, and the local ethics committee, in accordance with the Helsinki Declaration: CER-VD 2020-02204 for studies using skin samples, and CER-VD 2020-01257 for studies using post-mortem lungs. For COVID-19 skin samples, 10 consecutive patients presenting with moderate-to-severe COVID-19 disease and associated skin manifestations, hospitalized at CHUV from the beginning of the COVID pandemic in March 2020 were selected. All patients had positive PCR tests from nasal swab for SARS-CoV-2 and a clinical diagnosis of COVID-19. Comprehensive information on comorbidities, immunosuppressive treatment, type of skin manifestation and time from first symptoms are given in Extended Data Fig. $1 \mathrm{~b}$. In addition, the maximal disease severity score, determined according to the NIH ordinal scale and Sequential Organ Failure Assessment (SOFA) is provided. The score is defined as (1) not hospitalized with no limitation of activities; (2) not hospitalized with limitation of activities and/or home oxygen requirement; (3) hospitalized but not requiring supplemental oxygen and no longer requiring ongoing medical care; (4) hospitalized and not requiring supplemental oxygen but requiring ongoing medical care; (5) hospitalized requiring supplemental oxygen; (6) hospitalized requiring non-invasive ventilation or the use of high-flow oxygen devices; (7) hospitalized receiving invasive mechanical ventilation or extracorporeal membrane oxygenation; and (8) death. Control skin samples included skin lesions from $\operatorname{CLE}(n=11)$, plaque-type psoriasis $(n=21)$, atopic dermatitis $(n=16)$, lichen planus $(n=5)$ and healthy skin $(n=4)$. All patient biopsies were taken after informed consent was obtained and clinical diagnosis of the disease was histologically confirmed.

For the lung studies, eight post-mortem examinations of patients who died from COVID-19 at CHUV since March 2020 were included. All patients had positive PCR-tests from nasal swabs for SARS-CoV-2 and a clinical diagnosis of COVID-19. Patient information including comorbidities, immunosuppressive treatment, type of DAD, duration of symptoms until death and days of mechanical ventilation are given in Extended Data Fig. 6 and in Berezowska et al..$^{25}$.

\section{Assessment of the endotheliopathy index in skin lesions}

For scoring the endotheliopathy in skin samples, the outer and inner diameters of the post-capillary vessels of the superficial and middle dermis were measured and the endothelial swelling index was calculated as the ratio of the two diameters.

\section{Mice}

Twelve-to-sixteen-week-old female K18-hACE C57BL/6J transgenic mice (strain:2B6.Cg-Tg(K18-ACE2)2PrImn/J) were obtained from The Jackson Laboratory. Mice were housed in groups and fed standard chow diets. Mice were housed in groups of up to five mice per cage at $18{ }^{\circ} \mathrm{C}-24^{\circ} \mathrm{C}$ ambient temperature with 40-60\% humidity. Mice were maintained on a 12-h light-dark cycle from 06:00 to 18:00. Food and water were available ad libitum. Mice were administered $1 \times 10^{4} \mathrm{PFU}$ of SARS-CoV-2 by intranasal administration. Virus inoculations were performed under anaesthesia that was induced and maintained with ketamine hydrochloride and midazolam, and all efforts were made to minimize animal suffering. The cages were assigned randomly for vehicle and $\mathrm{H}-151$ treatment groups after the SARS-CoV-2 infection, and the experimenter was not blinded afterwards. Mice intraperitoneally received daily either dimethyl sulfoxide (DMSO) as vehicle or $750 \mathrm{nmol} \mathrm{H-151}$ in $200 \mu \mathrm{l} \mathrm{PBS5 \%} \mathrm{Tween-80.}$ Mice were euthanized on the indicated day and immediately dissected for transcardial perfusion with $20 \mathrm{ml}$ ice-cold PBS. Lungs and brains were collected. Half of each lung lobe was fixed in $4 \%$ PFA for histological analysis, and the other half of the lobes was chopped and stored for further analysis. For the survival study, mice were administered SARS-CoV-2 by intranasal administration as described above. Mice were euthanized when they reach one of the humane end point criteria:(1) more than $25 \%$ weight loss; (2) paralysis; (3) severe dyspnea. Animal experiments were approved by the Service de la Consommation et des Affaires Vétérinaires of the canton of Vaud (Switzerland) and were performed in accordance with the respective legal regulations.

\section{Plaque-forming assay}

Lung and brain of the mice were homogenized in Vero-E6 cell-culture medium $(\mathrm{DMEM}+10 \% \mathrm{FBS}+\mathrm{P} / \mathrm{S})$. Homogenized mix was centrifuged at $400 \mathrm{~g}$ for $10 \mathrm{~min}$. The supernatant was analysed for the viral content. Vero-E6 cells were seeded in a 12 -well plate at a density of $2.5 \times 10^{5}$ cells per well. Cells were washed with PBS and inoculated with viruses serially diluted in cell-culture medium. One hour after the infection, cells were washed with PBS, and overlaid with $0.8 \%$ Avicel (GP 3515) mixed at 1:1 with DMEM supplemented with $4 \%$ fetal bovine serum, 200 units $\mathrm{ml}^{-1}$ penicillin and $200 \mathrm{\mu g} \mathrm{ml}^{-1}$ streptomycin. After $72 \mathrm{~h}$ of incubation, the overlay was removed and cells were washed with PBS, fixed with $4 \%$ PFA and stained with crystal violet.

\section{Immunofluorescence and immunohistochemistry analysis}

Formalin-fixed paraffin-embedded (FFPE) skin blocks were cut into 6- $\mu \mathrm{m}$ sections and placed on slides. Sections were first deparaffinized and rehydrated, then Heat-Induced Epitope Retrieval (HIER) was performed and sections were permeabilized with PBS 0.01\% Triton. Samples were stained with primary antibodies (Supplementary Table 1) for 2 hours at room temperature. For immunofluorescence analysis, sections were then stained with fluorescently labelled secondary antibodies (Supplementary Table 1) for $30 \mathrm{~min}$ at room temperature. For immunohistochemistry, sections were stained with HRP-conjugated secondary antibodies followed by DAB staining and Mayer counterstaining. For RNA fluorescence in situ hybridization (FISH), IFNB1 mRNA was detected in skin using RNAScope Multiplex Fluorescent V2 Assay following the manufacturer's instructions (Advanced Cell Diagnostics, Inc.). Co-staining of sections with mouse anti-human CD163 (Diagnostic Bio Systems) was performed as described above. Images were acquired with a Zeiss LSM 700 confocal microscope and analysed with Zen 2010 software. For cell quantification, slides were digitalized using the PANNORAMIC 250 Flash digital scanner (3DHISTECHLtd) and cell types were quantified using the QuantCenter plug-in 2.2 of Caseviewer 2.4 software.

For LoC samples, the fixed LoCs were permeabilized with $0.1 \%$ Triton, $2 \%$ saponin and incubated with a blocking solution of $2 \%$ bovine serum albumin (BSA) for $1 \mathrm{~h}$ followed by overnight incubation with the primary antibody (1:100 dilution) in the blocking buffer at $4{ }^{\circ} \mathrm{C}$. The chip was then incubated with secondary antibodies (1:300 dilution) for $1 \mathrm{~h}$ at room temperature. A list of the primary antibodies and concentrations used is included in Supplementary Table 1. F-actin was stained using Sir-Actin dye in the far-red (Spirochrome) at $1 \mu \mathrm{M}$ for 30 min concurrently with Hoechst staining.

Mouse lungs were cut into $3-\mu \mathrm{m}$ sections. The extent of lung inflammation was quantified as the average percentage of lung surface area in which the alveolar wall is thickened with at least $50 \%$ decreased airspace area and was assessed by two independent investigators using three lung sections per mouse. TUNEL staining was performed using a commercially available kit (Promega) according to the manufacturer's instructions. Imaging was performed using the Zeiss Axioplan fluorescence microscope with the use of Axiovision software. Three fields were selected randomly from each lung piece. TUNEL-positive cells were quantified by automated counting performed by image analysis software (ImageJ).

\section{RNA extraction}

Excised 4-mm skin biopsies were immediately snap-frozen in liquid nitrogen and stored at $-80^{\circ} \mathrm{C}$ until processing. RNA was isolated using the TRIzol/chloroform method and a tissue homogenizer (Thermo Fisher Scientific). All isolated RNA had an A260/A280 value $\geq 1.7$ and 
RNA integrity was analysed on a Fragment analyser (Agilent). Mouse lung pieces were lysed in TRIzol (Thermo Fisher Scientific) and RNA was isolated according to the manufacturer's instructions. RNA from cells in lung-on-chip experiments was isolated by using the RNeasy Micro Kit (Qiagen) according to the manufacturer's instructions.

\section{NanoString analysis}

mRNA expression of 600 targets was analysed with the nCounter Human Immunology V2 panel including 20 customized probes (Nanostring Technologies, Seattle, WA, USA) on the nCounter platform (Nanostring Technologies) using 100 ng of RNA per skin sample. This commercial panel was extensively validated in-house for accuracy, repeatability and reproducibility before analysing the study samples. A quality check was run for each sample before including it into the analysis. Data were normalized and analysed using either nSolver 4.0 (Nanostring Technologies) or ROSALIND (ROSALIND Inc., San Diego, CA). Housekeeping probes to be used for normalization are selected based on the geNorm algorithm as implemented in the NormqPCR R library ${ }^{49}$. Clustering of genes for the final heatmap of differentially expressed genes was done using the PAM (Partitioning Around Medoids) method using the fpc R library (https://cran.r-project.org/web/packages/fpc/index.html) that takes into consideration the direction and type of all signals on a pathway and the position, role and type of every gene. The $z$-scores of each gene were then calculated for the selected patients to generate heatmaps and determine specific classifiers.

\section{STING inhibition in skin explants}

Six-millimetre skin biopsies from healthy individuals or patients with COVID-19 were cut into three equal pieces and one piece was snap-frozen to measure the baseline gene expression. The two remaining pieces were cultured in $200 \mu$ l of DMEM $10 \%$ FBS, $1 \%$ penicillinstreptomycin in the presence or not of $0.5 \mu \mathrm{g} \mathrm{ml}^{-1}$ of $\mathrm{H}-151$ for $15 \mathrm{~h}$. Skin biopsies were then homogenized in TRIzol to perform RNA extraction followed by NanoString analysis as described above.

\section{2'3'-cGAMP enzyme-linked immunosorbent assay}

Six-millimetre skin punch biopsies were lysed in Pierce RIPA Buffer using a tissue homogenizer (Thermo Fisher Scientific). Protease inhibitor cocktail (Sigma) was added to prevent protein degradation. Thirty micrograms of the lysate was used to measure 2'3'-cGAMP concentrations by enzyme-linked immunosorbent assay (ELISA) and according to the manufacturer's instructions (Cayman Chemical).

\section{Ultrastructural analysis of the skin}

For transmission electron microscopy, the skin biopsies were fixed in $2 \%$ glutaraldehyde in $0.1 \mathrm{M}$ sodium cacodylate buffer, $\mathrm{pH}$ 7.4. Samples were then post-fixed in $1 \% \mathrm{OsO}_{4} / 1.5 \%$ potassium ferrocyanide in aqua bidest for $2 \mathrm{~h}$, block stained with uranyl acetate ( $2 \%$ in distilled water), dehydrated in alcohol (stepwise 50-100\%), immersed in propylenoxide and embedded in glycidyl ether (polymerized $48 \mathrm{~h}$ at $60^{\circ} \mathrm{C}$; SERVA, Electrophoresis $\mathrm{GmbH}$, Heidelberg, Germany). Semi-thin and ultra-thin sections were cut with an ultramicrotome (Ultracut, Reichert Inc., Vienna, Austria). Ultra-thin sections ( $30 \mathrm{~nm}$ ) were mounted on copper grids and analysed on a Zeiss LIBRA 120 transmission electron microscope (Carl Zeiss, Oberkochen, Germany) operating at $120 \mathrm{kV}$.

\section{RT-qPCR analysis}

For mouse lung samples, RNA was reverse-transcribed using the RevertAid First Strand cDNA Synthesis reagents (Thermo Fisher Scientific), and quantitative PCR with reverse transcription (RT-qPCR) was performed in duplicate using Maxima SYBR Green Master Mix (Thermo Fisher Scientific) on QuantStudio 6/7 qPCR instruments. For LoC samples, RNA was reverse-transcribed using the SuperScript IV First-Strand Synthesis System with random hexamers (Invitrogen), and RT-qPCR reactions were prepared with SYBR Green PCR Master Mix (Applied
Biosystems) on the ABI PRISM 7900HT System (Applied Biosystems). Amplicon specificity was confirmed by melting-curve analysis. The primer sequences are listed in Supplementary Table 2.

\section{Immunoblotting}

SDS-loading buffer was mixed with the lung lysates in RIPA buffer and denatured at $95^{\circ} \mathrm{C}$ for 10 min. Lysates were separated by $10 \%$ SDS-PAGE and transferred onto nitrocellulose membranes. Blots were incubated with anti-p-p65 (Ser468) (1:1,000 dilution), and anti-p-STAT1 (Tyr 701) (1:1,000 dilution) (Cell Signaling) and anti- $\beta$-actin-HRP (1:2,000 dilution) (Santa Cruz Biotechnology). Proteins were visualized with the enhanced chemiluminescence substrate ECL (Pierce, Thermo Fisher Scientific) and imaged using the ChemiDoc XRS Biorad Imager and Image Lab Software 5.1. Uncropped images are presented in Supplementary Fig. 1.

\section{Primary human cell culture and culture of macrophage cell lines} Primary human alveolar epithelial cells (epithelial cells) and human lung microvascular endothelial cells (endothelial cells) were obtained from a commercial supplier (Cell Biologics). All chips were reconstituted with epithelial cells seeded directly on the LoC without any additional in vitro culture. Endothelial cells were passaged 3-5 times before seeding in the LoC devices. Experiments were performed with cells from at least two donors.

Peripheral blood mononuclear cells from buffy coat (Interregional Blood Transfusion SRC) were obtained from anonymized donors and isolated using a Biocol Separation procedure as per the manufacturer's instructions. One week before seeding the macrophages in the LoC devices, a cryopreserved aliquot was cultured in a T-75 flask (TPP) in RPMI supplemented with $10 \%$ FBS. CD14 ${ }^{+}$monocytes were isolated using positive selection (CD14 ultrapure isolation kit, Miltenyi Biosciences), embedded in hemispherical domes of basement membrane extract (BME-2, Cultrex) in 24-well plates, and cultured in RPMI medium supplemented with $10 \% \mathrm{FBS}, 20 \mathrm{ng} \mathrm{ml}^{-1}$ recombinant human macrophage-colony stimulating factor protein (M-CSF) and $100 \mathrm{U} \mathrm{I}^{-1}$ of penicillin-streptomycin solution (Thermo Fisher Scientific). The monocytes were differentiated for 7 days. On the day of seeding into the LoC, the BME domes were first disrupted by scraping with a $\mathrm{P} 1000$ pipette. The mechanically dissociated hydrogel was then brought to semi-liquid state by adding $500 \mu \mathrm{l}$ of ice cold RPMI medium per well. The BME-RPMI suspension was then centrifuged at $200 \mathrm{~g}$ for $5 \mathrm{~min}$ in a $15-\mathrm{ml}$ Falcon tube pre-coated with 1\% BSA in PBS and resuspended in 4-5 ml of ice-cold Cell Recovery Solution (Corning) over ice for 20-30 min. Occasionally, the solution was sheared with a fire-polished glass pipette to ensure the complete removal of the macrophages from the BME hydrogel. The cell suspension was then washed twice with $10 \mathrm{ml}$ of RPMI medium/10\% FBS to remove remaining traces of the cell recovery reagent. If required, BME-2 suspension was sometimes incubated in 2-3 ml trypsin to remove remaining fragments of BME-2, and the washing step was repeated again. Isolated macrophages were resuspended in epithelial cell medium and passed through a $40-\mu \mathrm{m}$ filter to obtain a single-cell suspension of macrophages.

Wild-type and $c G A S^{-/-}$THP-1 cells were cultured according to the suppliers' instructions. THP-1 cells were differentiated with $5 \mathrm{ng} \mathrm{ml}^{-1} \mathrm{PMA}$ for 3 days and transferred to the vascular channel of the LoC at $2 \mathrm{dpi}$.

\section{Production of lentiviral vectors and transduction of primary epithelial and endothelial cells}

HEK-293T cells were a gift from the laboratory of D. Trono. HEK-293T cells were transfected with pCMVDR8.74, pMD2.G plasmids and the puromycin-selectable pLKO.1-puro lentiviral vector containing the shRNA for human STING (5'-CATGGTCATATTACATCGGAT-3') and human MAVS ( $5^{\prime}$-CAAGTTGCCAACTAGCTCAAA- $3^{\prime}$ ) by the calcium phosphate precipitation method. The supernatant containing lentiviral particles was collected at 48 and $72 \mathrm{~h}$, pooled and concentrated by ultracentrifugation. Primary endothelial cells (shRNA for STING and MAVS) and primary alveolar epithelial cells at passage 5 (shRNA 
for STING only) were transduced with the lentiviral vectors by directly adding $10 \mu \mathrm{l}$ to the culture medium; transduced cells were selected by adding $1 \mu \mathrm{g} \mathrm{ml}^{-1}$ puromycin to the medium $48 \mathrm{~h}$ after the transduction.

\section{Generation of SARS-CoV-2 stocks}

VeroE6 cells and a clinical isolate of SARS-CoV-2 were a gift from the laboratory of C. Tapparel.SARS-CoV2/Switzerland/GE9586/2020 was isolated from a clinical specimen in the University Hospital in Geneva in Vero-E6 cells. Vero-E6 cells were infected and supernatant was collected at $3 \mathrm{dpi}$, clarified, aliquoted and frozen at $-80^{\circ} \mathrm{C}$ and subsequently titrated by plaque assay in Vero-E6. Viruses used for the LoC and animal experiments in this manuscript were at passage 2 and passage 4 , respectively, in Vero-E6 cells.

\section{Infection of the LoC model with SARS-CoV-2}

LoC devices were purchased from a commercial vendor (Emulate). For a small subset of experiments for RT-qPCR measurements in uninfected controls, devices fabricated in-house with similar dimensions (but without a stretching channel) using porous membranes supplied by a commercial vendor were used (BiOND). A detailed protocol for the establishment of the LoC model has been described previously ${ }^{24}$. In brief, devices were coated with ECM solution of $150 \mathrm{\mu g} \mathrm{ml}^{-1}$ bovine collagen type I (AteloCell,Japan) and $30 \mathrm{\mu g} \mathrm{ml}^{-1}$ fibronectin from human plasma (Sigma-Aldrich). For the three-component model with primary macrophages, differentiated primary human macrophages were seeded directly on the PDMS membrane $1-2 \mathrm{~h}$ before seeding of the endothelial cells on the basolateral side of the membrane and epithelial cells on the apical side. For experiments with $\rho^{0}$ endothelial cells, the endothelial cells were incubated with ddC for $3-5$ days before infection. The chip was incubated overnight with complete epithelial and endothelial medium in the respective channels under static conditions. Thereafter, a reduced medium for the air-liquid interface (ALI) was flowed through the vascular channel and the epithelial face was incubated with epithelial base medium supplemented with $1 \mu \mathrm{M}$ dexamethasone(Sigma-Aldrich). This medium was replaced daily for the following 2-3 days. Thereafter, the chips were maintained overnight at an ALI and then then transferred to the biosafety level 3 (BSL-3) facility for SARS-CoV-2 infection. Here, an aliquot of virus-containing supernatant was diluted approximately 20-fold in epithelial cell medium without FBS to generate the inoculum that corresponded to an infectious dose of 400-600 PFU in a volume of $30 \mu \mathrm{l}$. This volume was then added to the apical channel of each LoC, and the LoC was incubated for an hour at $37^{\circ} \mathrm{C}$ and $5 \% \mathrm{CO}_{2}$. Thereafter, the LoC was returned to ALI. For LoCs treated with the STING or the VDAC oligomerization inhibitor, $\mathrm{H}-151(1 \mu \mathrm{M})$ or VBIT-4 $(1 \mu \mathrm{M})$ was perfused through the vascular channel after infection and maintained over the course of 3 days. Infection was terminated at specified time points and the LoCs processed for RNA extraction or by fixation with freshly prepared $4 \%$ paraformaldehyde for a period of $30 \mathrm{~min}$.

\section{Confocal imaging and image analysis of LoCs}

Infected and control LoCs were imaged using a Leica SP8 confocal microscope with a white light laser. LoCs were imaged with a $25 \times$ water immersion objective (NA $=0.95$, Leica), with standard settings (voxel size $227.27 \times 227.27 \times 300 \mathrm{~nm}^{3}$ ) across chips labelled the same way. $Z$-stacks were subsequently deconvolved using the Huygens Deconvolution Software (Scientific Volume Imaging) and 3D views were rendered using Imaris (Bitplane). Maximum intensity projects were rendered using ImageJ. The following parameters were used for generation of the surfaces in Imaris for the visualization of IFN $\beta$, cleaved caspase-3, macrophages and $\mathrm{p}$-STING. In each case, uninfected control chips and/ or infected chips and/or treated chips from the same experiment were immunostained and imaged together, to control for differences in the immunofluorescence intensities across antibody aliquots, imaging conditions, and microscopes. Chips from the same experiment were analysed using the same Imaris parameters. Three-cell component chips in Fig. 3b, Extended Data Fig. 6b, IFN $\beta$ : manual threshold: 110, smoothing: $0.455 \mu \mathrm{m}$. Three-cell component chips in Extended Data Fig. 6d, IFN $\beta$ : manual threshold: 110 , smoothing: $0.455 \mu \mathrm{m}$. Two-cell component chips in Fig. 3c, Extended Data Fig. 7b, IFN $\beta$ : manual threshold: 45, smoothing: $0.455 \mu \mathrm{m}$. Three-cell component chips in Extended Data Fig. 6e, cleaved caspase-3: manual threshold: 110, smoothing: $0.8 \mu \mathrm{m}$. Two-cell component chips in Fig. $3 \mathrm{c}$, cleaved caspase-3: manual threshold: 110 , smoothing: $0.8 \mu \mathrm{m}$. Two-cell component chips in Fig. 3d., p-STING: manual threshold:110, smoothing: $0.8 \mu \mathrm{m}$. Three-cell component chips in Fig 3b, CD45: manual threshold: 110, smoothing: $1 \mu \mathrm{m}$. Two-cell component chips in Fig. $3 \mathrm{~g}$, Extended Data Fig. $8 \mathrm{~d}$, IFN $\beta$; manual threshold: 30 ; smoothing: $0.455 \mu \mathrm{m}$.

\section{Sample preparation for proteomic analysis}

Cells from the vascular and apical faces of the LoC devices were extracted in a sequential manner by instillation of $0.25 \%$ Trypsin-EDTA solution (Gibco) in the vascular channel followed by the apical channel. Cells were centrifuged at $300 \mathrm{~g}$ for $5 \mathrm{~min}$ and washed twice with PBS solution to eliminate extracellular matrix components. Pelleted cells were then resuspended in a $20-\mu$ l solution of $100 \mathrm{mM} \mathrm{HEPES} \mathrm{pH} 8$ and $5 \mathrm{mM}$ tris(2-carboxyethyl)phosphine and heat-inactivated at $95^{\circ} \mathrm{C}$ for 10 min before removal from the BSL-3 facility, and stored at $-20^{\circ} \mathrm{C}$ for subsequent processing at the Proteomics Core Facility. Here cells were vacuum-centrifuged to near dryness and resuspended in $9 \mu \mathrm{l}$ of $100 \mathrm{mM}$ HEPES pH 8 and $10 \mathrm{mM}$ tris(2-carboxyethyl)phosphine. Samples were first heated for $20 \mathrm{~min}$ at $95^{\circ} \mathrm{C}$ with permanent shaking and then sonicated in a water bath for $15 \mathrm{~min}$. Extracted proteins were alkylated with $1 \mu \mathrm{l}$ of $400 \mathrm{mM}$ chloroacetamide for $30 \mathrm{~min}$ at $37^{\circ} \mathrm{C}$ in the dark with permanent shaking. Proteins were digested overnight using $400 \mathrm{ng}$ mass spectrometry grade trypsin with permanent shaking. The resulting peptides were desalted on C18 StageTips ${ }^{50}$ and dried by vacuum centrifugation. Peptides were reconstituted in $8 \mu$ I HEPES $100 \mathrm{mM}$ $\mathrm{pH} 8$ and labelled with $3 \mu \mathrm{l}$ of isobaric tags (TMT $20 \mu \mathrm{g} \mu \mathrm{l}^{-1}$ in pure acetonitrile) for $90 \mathrm{~min}$ at room temperature. The labelling reaction was stopped with addition of $50 \%$ hydroxylamine (final concentration $0.4 \%$ $(\mathrm{v} / \mathrm{v})$ ) for $15 \mathrm{~min}$. A small fraction of the labelled peptides was mixed in a 1:1 ratio across all samples and analysed with a single shot control liquid chromatography-tandem mass spectrometry (LC-MS/MS) run to evaluate the mixing accuracy. On the basis of the results of this control run, the remaining labelled peptides were mixed in equal amounts, vacuum-centrifuged and fractionated into eight fractions using the Pierce High pH Reversed-Phase Peptide Fractionation Kit following the manufacturer's instructions. The eight fractions were dried by vacuum centrifugation and stored at $-20^{\circ} \mathrm{C}$.

\section{Mass spectrometry}

Peptides were resuspended in 2\% acetonitrile, $0.1 \%$ FA and analysed on a Lumos Fusion Orbitrap Mass Spectrometer online connected to a Dionex Ultimate 3000 RSLC nano UPLC system. A capillary precolumn (Acclaim Pepmap C18, $3 \mu \mathrm{m} 100 \AA, 2 \mathrm{~cm} \times 75 \mu \mathrm{m}$ inner diameter) was used for sample trapping and cleaning. Analytical separations were performed at $250 \mathrm{nl} \mathrm{min}{ }^{-1}$ over 150-min biphasic gradients on a 50-cm-long in-house packed capillary column (75 $\mu$ m inner diameter, ReproSil-Pur C18-AQ1.9 $\mu \mathrm{m}$ silica beads, Dr. Maisch). Acquisitions were performed through the top speed data-dependent acquisition mode using a 3 s cycle time. First MS scans were acquired at a resolution of $120,000($ at $200 \mathrm{~m} / \mathrm{z}$ ) and the most intense parent ions were selected and fragmented by high energy collision dissociation (HCD) with a normalized collision energy (NCE) of $37.5 \%$ using an isolation window of $0.7 \mathrm{~m} / \mathrm{z}$. Fragmented ion scans were acquired with a resolution of $50,000($ at $200 \mathrm{~m} / \mathrm{z}$ ) and selected ions were then excluded for the following 120 s.

\section{Mass spectrometry data analysis}

Raw data were processed using SEQUEST, Mascot, MS Amanda ${ }^{51}$ and MS Fragger $^{52}$ in Proteome Discoverer v.2.4 against a concatenated database 
consisting of the Uniprot human reference proteome (release 2020_10) and UniprotSARS-CoV-2 reference proteome (release 2020_10). Enzyme specificity was set to trypsin and a minimum of six amino acids was required for peptide identification. Up to two missed cleavages were allowed and a $1 \%$ false discovery rate (FDR) cut-off was applied both at peptide and at protein identification levels. For the database search, carbamidomethylation $(\mathrm{C})$ and TMT tags ( $\mathrm{K}$ and peptide $\mathrm{N}$ termini) were set as fixed modifications whereas oxidation (M) was considered as a variable. The resulting text files were processed through in-house written R scripts (v.3.6.3). Two steps of normalization were applied: sample loading (SL) and trimmed mean of M-values (TMM) normalization. The SL normalization ${ }^{53}$ assumes that total protein abundances are equal across the TMT channels; therefore, the reporter ion intensities of all spectra were summed and each channel was scaled according to this sum, so that the sum of reporter ion signals per channel equals the average of the signals across samples. Subsequently, the TMM normalization step was applied using the package EdgeR (v.3.26.8) ${ }^{54}$. This normalization step works on the assumption that most of the protein abundances do not change across samples therefore, it calculates normalization factors according to these presumed unchanged protein abundances. Differential protein expression analysis was performed using the R bioconductor package limma (v.3.40.6, 2020-02$29)^{55}$, followed by the Benjamini-Hochberg multiple-testing method ${ }^{56}$. $P$ values lower than $0.00128($ FDR $<0.05)$ and absolute $\log _{2}$-transformed fold change $\left(\log _{2} \mathrm{FC}\right)>0.5$ were considered as significant. For the time-course study, all quantified proteins were monitored. The significant temporal dynamics were defined with the timecourse package in R Bioconductor, which uses a multivariate empirical Bayes model to rank proteins ${ }^{57}$. Replicate time-course data can be compared allowing for variability both within and between time points. The mb.long method was used to calculate the moderated Hotelling $\mathrm{T}^{2}$ statistic, specifying a one-dimensional method (method = "1D"), in which significant proteins change over the time course. The null hypothesis is that the protein temporal profile is equal to 0 .

\section{Statistics and reproducibility}

Statistical analyses are described in each figure legend. For experiments combining several groups, an ordinary one-way ANOVA test was used. Statistical significance was determined using Prism v.8.0 software (GraphPad). Significant differences between groups were determined by post-hoc Tukey's multiple comparisons tests, unless specified otherwise, $P>0.05$ was considered non-significant. The Student's $t$-test or the Mann-Whitney test was used to assess the $P$ value when comparing only two groups. For LoC studies, fields of view from a given LoC are considered as biological replicates, and the number of LoCs corresponds to the number of times the experiment was repeated. Images of p-STING ${ }^{+}$ endothelial cells in Fig. $3 \mathrm{~d}$ are from $n=2$ fields of view from $n=1 \mathrm{LoC}$. Data of mitochondria with loss of cristae morphology are representative slices from volumetric electron microscopy imaging of $n=4$ endothelial cells from $n=2$ infected LoCs. Data from patient samples were obtained from $n=3$ independent experiments and quantifications for histological analysis were performed by $n=2$ independent investigators.

\section{Reporting summary}

Further information on research design is available in the Nature Research Reporting Summary linked to this paper.

\section{Data availability}

Full scans for all western blots and the in-gel fluorescence images are provided in Supplementary Fig. 1 and the limma and timecourse analyses of the proteomics data are provided in Supplementary Table 3. Raw data supporting the findings of this study have been deposited at Zenodo and are publicly available at https://doi.org/10.5281/ zenodo.5818157. The proteomics dataset generated during this study has been deposited in the PRIDE database with accession code PXD030753. The NanoString dataset generated during this study has been deposited at the Gene Expression Omnibus (GEO) database with accession code GSE193068. Source data are provided with this paper.

\section{Code availability}

Custom scripts in R for the analysis of proteomics data have been deposited at Zenodo and are publicly available at https://doi.org/10.5281/ zenodo.5818157.

49. Perkins, J. R. et al. ReadqPCR and NormqPCR: R packages for the reading, quality checking and normalisation of RT-qPCR quantification cycle (Cq) data. BMC Genomics 13, 296 (2012).

50. Rappsilber, J., Mann, M. \& Ishihama, Y. Protocol for micro-purification, enrichment, pre-fractionation and storage of peptides for proteomics using StageTips. Nat. Protoc. 2 1896-1906 (2007).

51. Dorfer, V. et al. MS Amanda, a universal identification algorithm optimized for high accuracy tandem mass spectra. J. Proteome Res. 13, 3679-3684 (2014).

52. Kong, A. T., Leprevost, F. V., Avtonomov, D. M., Mellacheruvu, D. \& Nesvizhskii, A. I. MSFragger: ultrafast and comprehensive peptide identification in mass spectrometrybased proteomics. Nat. Methods 14, 513-520 (2017).

53. Plubell, D. L. et al. Extended multiplexing of tandem mass tags (TMT) labeling reveals age and high fat diet specific proteome changes in mouse epididymal adipose tissue. Mol. Cell Proteomics 16, 873-890 (2017).

54. Robinson, M. D., McCarthy, D. J. \& Smyth, G. K. edgeR: a Bioconductor package for differential expression analysis of digital gene expression data. Bioinformatics 26, 139-140 (2010).

55. Ritchie, M. E. et al. limma powers differential expression analyses for RNA-sequencing and microarray studies. Nucleic Acids Res. 43, e47 (2015).

56. Klipper-Aurbach, Y. et al. Mathematical formulae for the prediction of the residual beta cell function during the first two years of disease in children and adolescents with insulin-dependent diabetes mellitus. Med. Hypotheses 45, 486-490 (1995).

57. Tai, Y. C. \& Speed, T. P. A multivariate empirical Bayes statistic for replicated microarray time course data. Ann. Statist. 34, 2387-2412 (2006).

Acknowledgements We thank the Genomic Technologies Facility (GTF) at University of Lausanne (UNIL) and the Immune Landscape Laboratory (Oncology department, CHUV) for accessing the NanoString platform; A. Joncic and I. Surbeck for technical assistance with the histological analysis; B. Fehrenbacher for technical assistance with transmission electron microscopy studies of COVID-19 skin samples; J. Blanc and G. Knott for technical assistance with serial block scanning electron microscopy of LoC devices and data analysis and volumetric reconstruction; R. Hamelin, F. Armand and M. Pavlou for technical assistance for the proteomic analysis studies in the LoC devices; F. Signorino-Gelo for LoC device fabrication; and N. Samson for help with BioRender. This work was supported by the COVID-19 National Research Program (NRP-78) to M.G. and A.A. (4078PO_198470) with additional support by the Leenaards Foundation to M.G. and A.A. and the Fondation Acteria and European Union's Horizon 2020 Research and Innovation program grant agreement (grant no. 804933, ImAgine) to A.A. V.V.T. received support from the Novartis Foundation for Medical-Biological Research (grant no. 20C240).

Author contributions J.D.D., F.S. and A.Y. conducted the experiments and associated analysis involving human tissues. A.Y., C.C. and M.G. selected patients and provided skin samples. C.v.G., L.d.L. and S.B. provided post-mortem lung samples. E.G. and M.S. performed electron microscopy analysis of COVID-19 skin lesions. V.V.T., M.F.G., K.S. and T.N. conducted in vitro LoC cell culture studies and the associated analysis. A.D. performed electron microscopy analysis of the LoC devices. M.F.G. performed the animal study and associated analysis and C.G. helped with the histopathological analysis. M.G. and A.A conceived and supervised the work and wrote the manuscript with comments from co-authors.

Competing interests A.A. is a scientific co-founder of IFM Due. The other authors declare no competing interests.

\section{Additional information}

Supplementary information The online version contains supplementary material available at https://doi.org/10.1038/s41586-022-04421-w.

Correspondence and requests for materials should be addressed to Michel Gilliet or Andrea Ablasser.

Peer review information Nature thanks Zhijian (James) Chen, Michael Matthay, Stanley Perlman and the other, anonymous, reviewer(s) for their contribution to the peer review of this work.

Reprints and permissions information is available at http://www.nature.com/reprints. 


\section{Article}

a
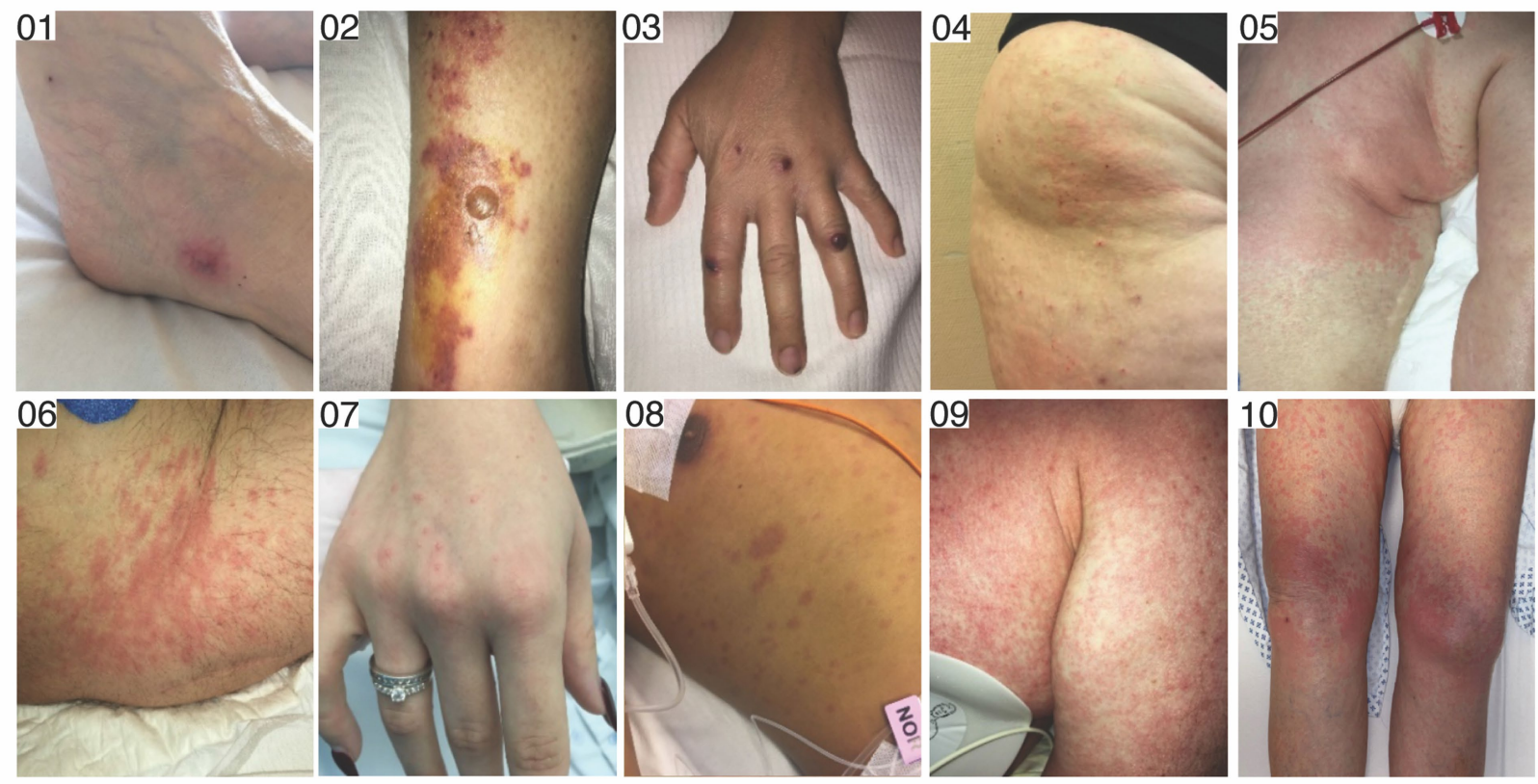

b

\begin{tabular}{|c|c|c|c|c|c|c|c|c|}
\hline ID & Sex & Age & Comorbidities & Cutaneous signs & $\begin{array}{l}\text { COVID-19 } \\
\text { severity }\end{array}$ & $\begin{array}{l}\text { Maximal } \\
\text { disease } \\
\text { severity }\end{array}$ & $\begin{array}{c}\text { SARS-CoV-2 } \\
\text { swab }\end{array}$ & $\begin{array}{l}\text { Time from } \\
\text { first symptoms } \\
\text { (days) }\end{array}$ \\
\hline 01 & female & 62 & Lung transplant, CKD & Purpuric-necrotic & Severe & 6 & Positive & 9 \\
\hline 02 & male & 65 & Kidney transplant, DM & Purpuric-necrotic & Critical & 8 & Positive & 10 \\
\hline 03 & male & 34 & Type 2 AlH & Purpuric-necrotic & Moderate & 4 & Positive & 4 \\
\hline 04 & female & 21 & none & Maculopapular & Moderate & 2 & Positive & 8 \\
\hline 05 & female & 62 & Lung transplant, COPD & Maculopapular & Severe & 7 & Positive & 17 \\
\hline 06 & male & 47 & none & Maculopapular & Severe & 7 & Positive & 18 \\
\hline 07 & female & 18 & none & Maculopapular & Moderate & 3 & Positive & 7 \\
\hline 08 & male & 22 & none & Maculopapular & Critical & 7 & Positive & 4 \\
\hline 09 & male & 56 & Overweight & Maculopapular & Severe & 7 & Positive & 11 \\
\hline 10 & female & 80 & Hypertension, HRD, dementia & Maculopapular & Severe & 5 & Positive & 13 \\
\hline
\end{tabular}

Extended Data Fig. 1 | Clinical characteristics of patients with COVID-19 with associated skin manifestations. a, Photographs of the skin lesions. b. Clinical parameters and demographics of the 10 patients with COVID-19 selected for this study. HRD, Heart Rhythm Disorder; Overweight (BMI $>25 \mathrm{~kg} /$ $\mathrm{m}^{2}$, but $<30 \mathrm{~kg} / \mathrm{m}^{2}$ ); COPD, chronic obstructive pulmonary disease; CKD, chronic kidney disease; DM, diabetes mellitus; $\mathrm{AlH}$, autoimmune hepatitis. 
a

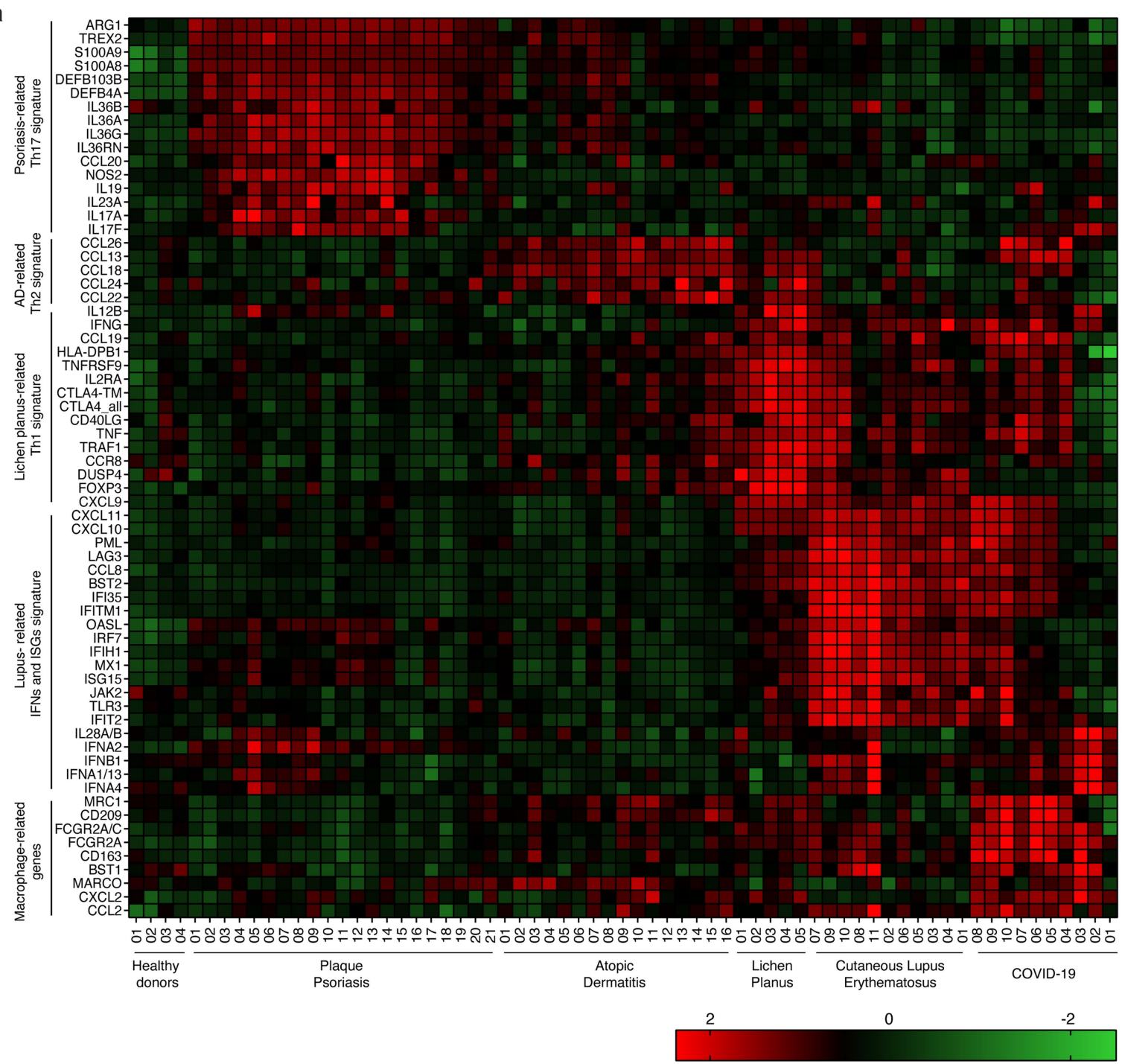

b

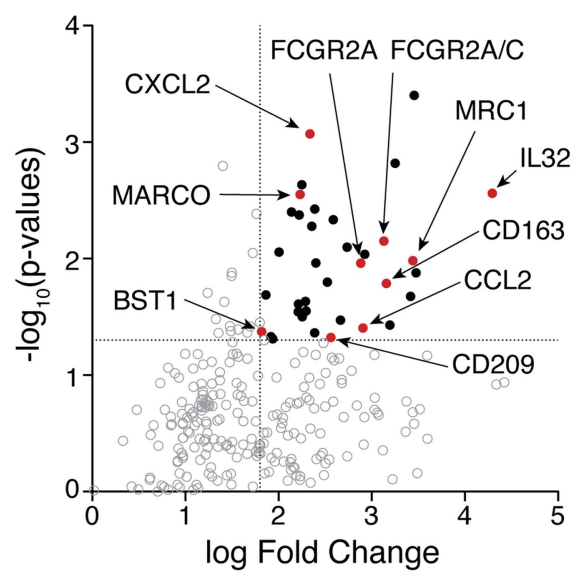

Extended Data Fig. 2 | Immune gene expression profiling of COVID-19 skin lesions and common inflammatory skin diseases. a, Immune gene expression profiles of patients with lichen planus $(n=5)$, cutaneous lupus erythematosus (CLE, $n=10)$, COVID-19 associated skin lesions $(n=10)$, plaque-type psoriasis $(n=21)$, and atopic dermatitis (AD, $n=16)$ compared to healthy skin (HD, $n=4)$ assessed by NanoString assay. Differentially expressed genes between different pairwise comparisons (i.e. each disease group vs other skin inflammatory diseases) were used to generate disease-related gene signatures. $P$ value $<0.05$, and fold change $>2$ were used as cutoffs to choose specific classifiers. b, Volcano plot of upregulated genes in COVID-19 compared with CLE skin lesions. 


\section{Article}

a
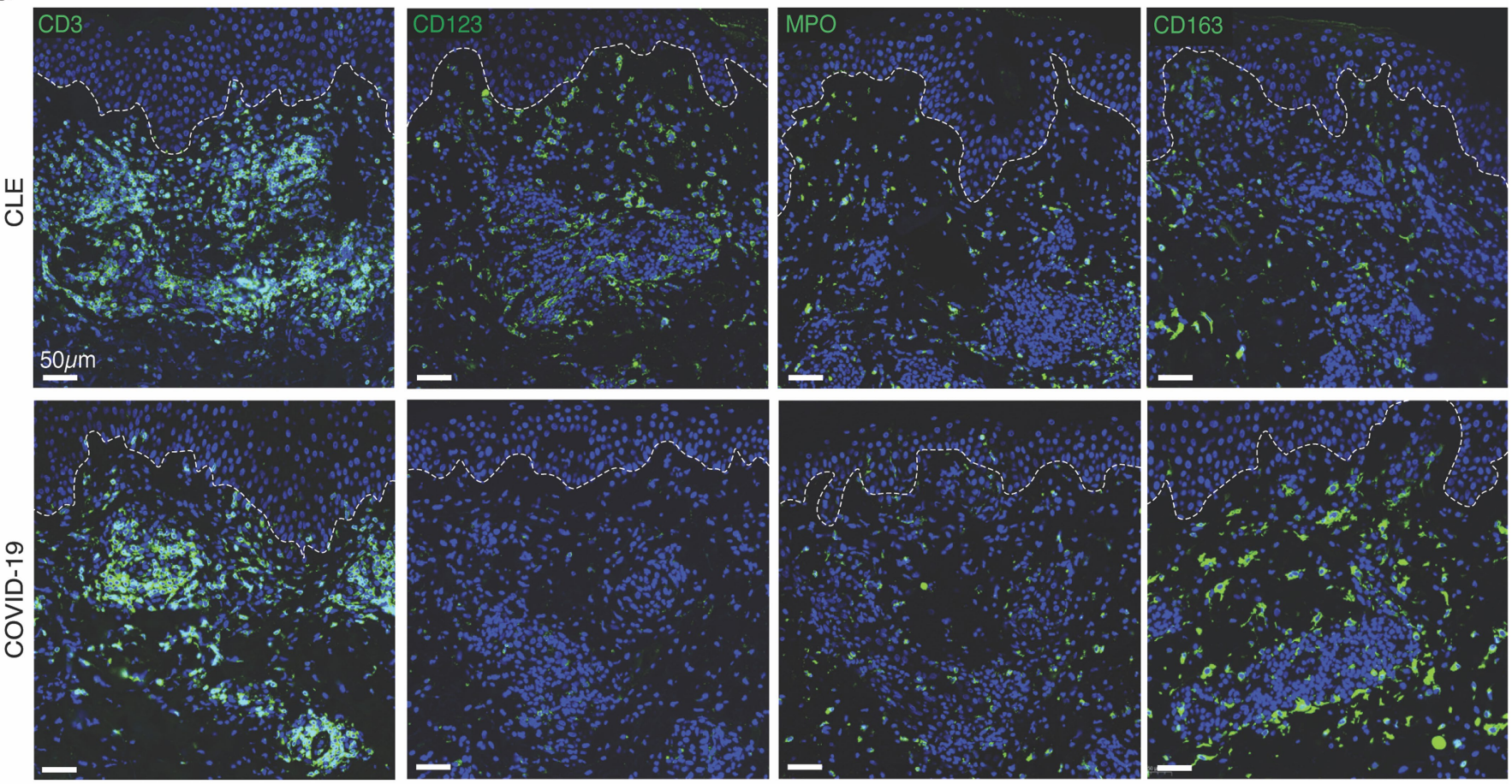

b

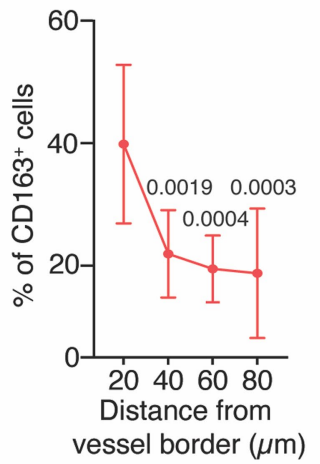

C

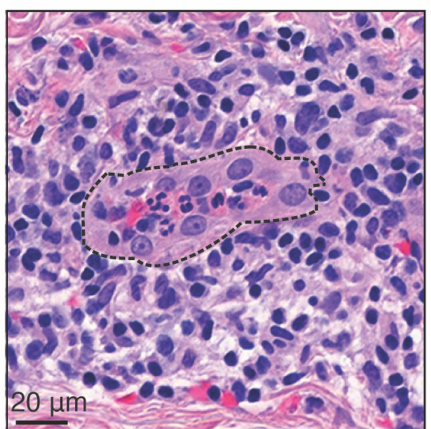

d

CLE

COVID-19

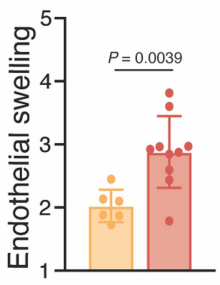

Extended Data Fig. 3 |Macrophages accumulate around vessels exhibiting prominent endotheliopathy in COVID-19 skin lesions. a, Confocal microscopy images of $\mathrm{CD}^{+} \mathrm{T}$ cells, $\mathrm{CD} 123^{+}$plasmacytoid dendritic cells, $\mathrm{MPO}^{+}$ neutrophils, and $\mathrm{CD}_{163^{+}}$macrophages in skin lesions from CLE (top row) and COVID-19 (bottom row). Images are representative of 10 patients with COVID-19 and 5 patients with CLE. b, Percentages of CD $163^{+}$macrophages present at different distances from blood vessel in COVID-19 skin lesions $(n=9) . c$, Representative histopathology image of a dermal blood vessel in COVID-19 skin lesions (H\&E stain). Blood vessel, dashed line. d, Endothelial cell swelling index, a measure of endotheliopathy, quantified in COVID-19 $(n=10)$ and CLE skin lesions $(n=6)$. P values were obtained with one-way ANOVA followed by Tukey's multiple comparison test (b) and two-tailed Student's $t$-test (d). 

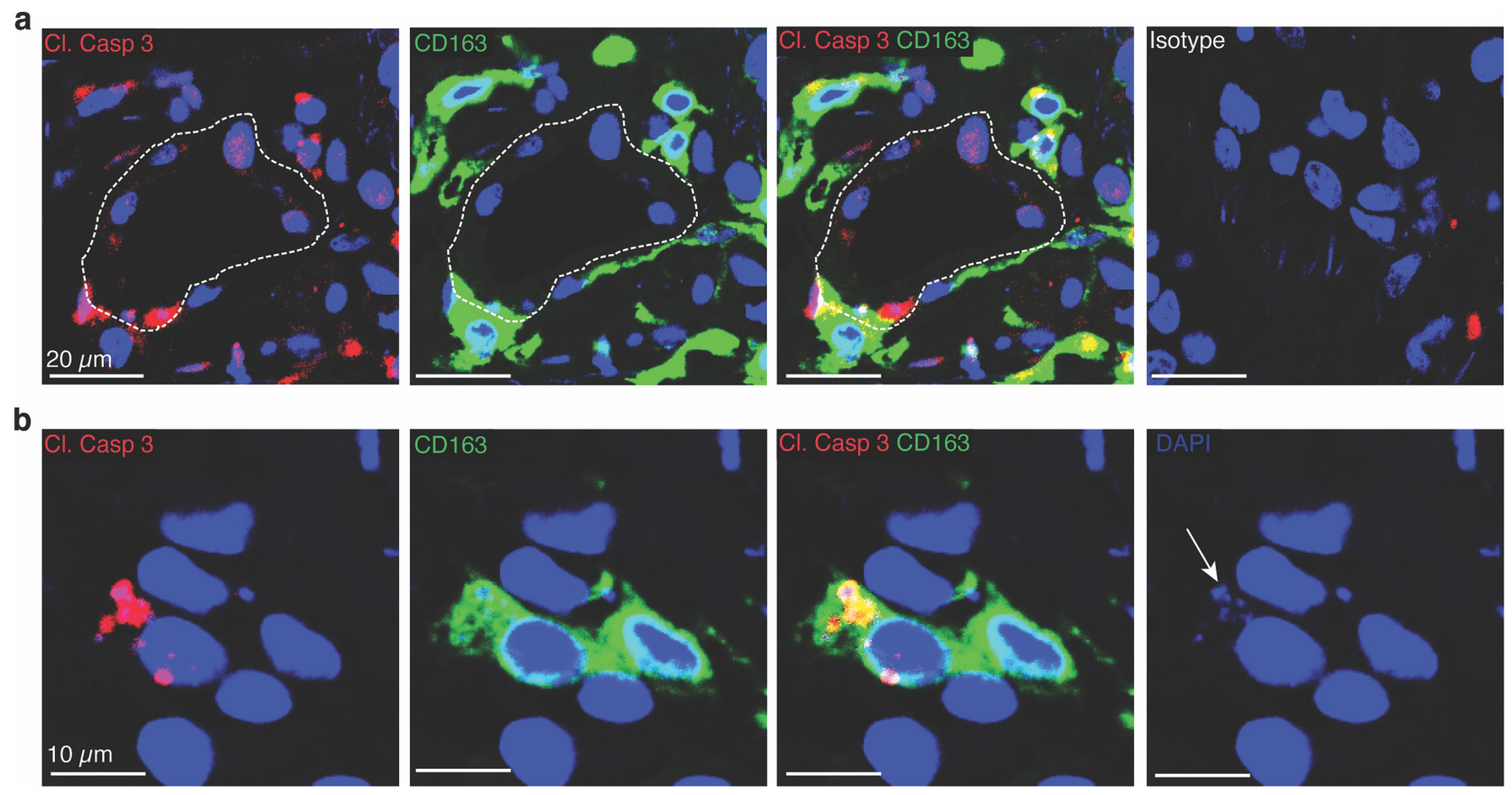

Extended Data Fig. 4 | Perivascular macrophages engulf dying cells. a, b, Confocal microscopy images of representative COVID-19 skin lesion stained for CD163 (green), cleaved caspase-3 (red) and DNA (DAPI). Images are representative of 10 patients with COVID-19. Blood vessel, dashed line. 


\section{Article}

a

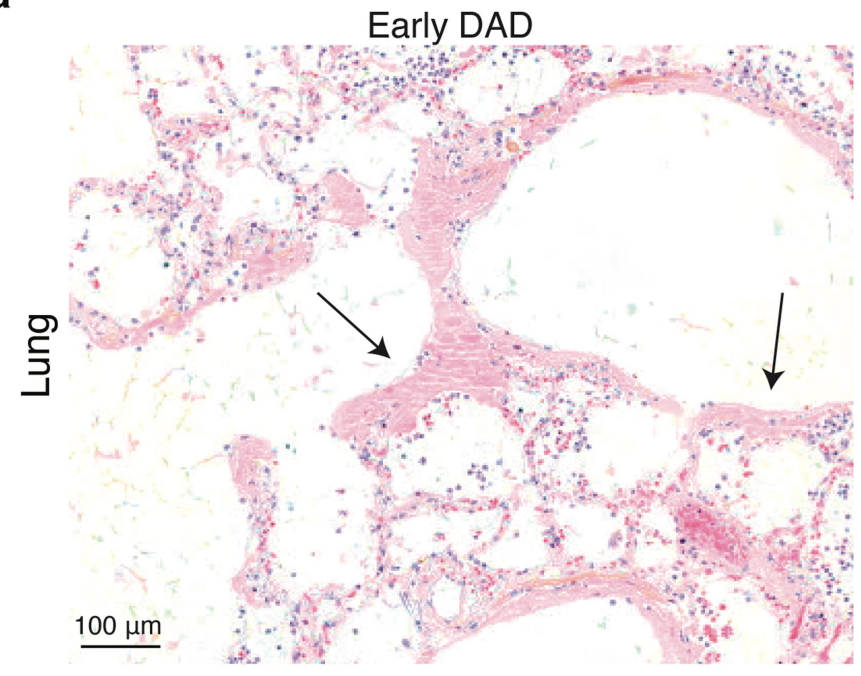

Late DAD



b

\begin{tabular}{|c|c|c|c|c|c|c|c|c|}
\hline Gender & Age & $\begin{array}{l}\text { Immuno- } \\
\text { suppressive } \\
\text { drugs }\end{array}$ & $\begin{array}{l}\text { Duration of symptoms } \\
\text { until death [duration of } \\
\text { mechanical ventilation] }\end{array}$ & DAD & $\begin{array}{c}\text { RT-qPCR } \\
\text { SARS-Cov2* }^{*}\end{array}$ & $\begin{array}{c}\text { IHC } \\
\text { SARS-Cov2** }^{*}\end{array}$ & $\begin{array}{r}\text { MxA } \\
(\%)\end{array}$ & $\begin{array}{c}\text { p-STING }{ }^{+} \\
\text {macrophages } \\
\text { /mm }^{2}\end{array}$ \\
\hline $\mathrm{F}$ & 72 & - & $6[-]$ & exudative & positive & positive & High (60) & 23 \\
\hline $\mathrm{F}$ & 72 & - & $8[-]$ & exudative & positive & positive & High (87) & 16 \\
\hline M & 96 & $\mathrm{HC}$ & $8[-]$ & exudative & positive & positive & High (73) & 15 \\
\hline M & 86 & - & $10[3]$ & exudative/proliferative & positive & positive & High (77) & 16 \\
\hline $\mathrm{F}$ & 71 & $\mathrm{HC}$ & $14[-]$ & exudative/proliferative & positive & positive & Low (17) & 4 \\
\hline M & 79 & $\mathrm{HC}$ & $16[4]$ & proliferative & negative & negative & Low (48) & 0 \\
\hline M & 75 & - & $17[5]$ & exudative/proliferative & negative & negative & Low (18) & 1 \\
\hline$M$ & 69 & $\mathrm{HC}$ & 38 [19] & proliferative & negative & negative & Low (15) & 0 \\
\hline
\end{tabular}

C

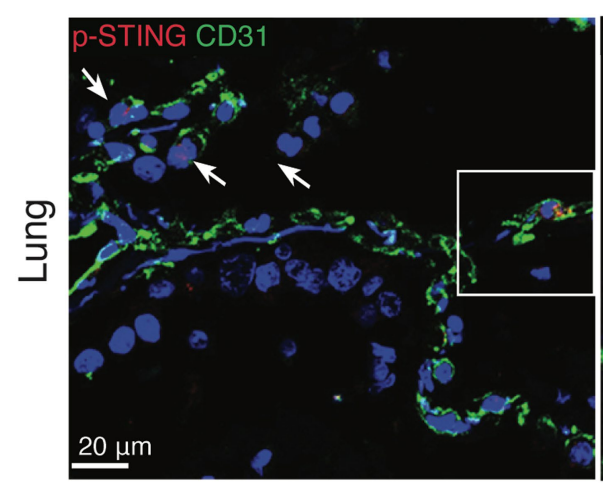

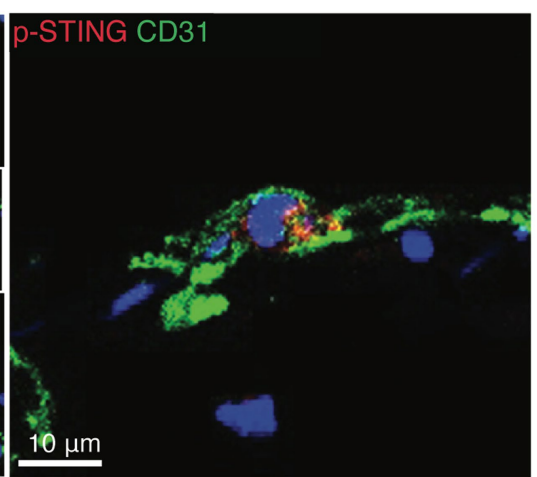

d

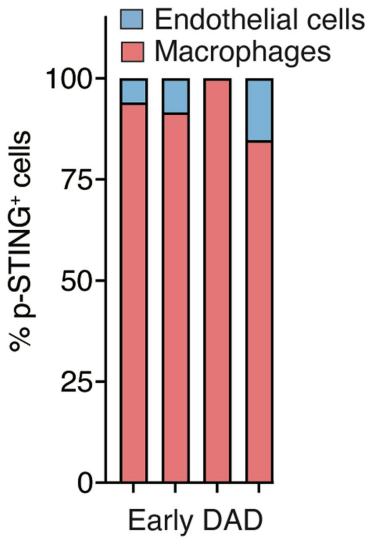

Extended Data Fig. 5 | Patient characteristics and histopathological analyses of a post-mortem COVID-19-affected lung. a, Representative histopathology image of a COVID-19 lung in the early phase of DAD with extensive hyaline membranes (left) or in the late phase of DAD with fibrosis obliterating the alveolar lumina (right) (H\&E stain). Arrows indicate hyaline membranes. b. Clinical parameters of the 8 patients with COVID-19 selected for the study. HC, hydroxychloroquine; Phase of the diffuse alveolar damage defined based on pure presence of hyaline membranes (exudative) or fibrotic changes (proliferative); ${ }^{*}$ limit of detection is 20.8 copies per reaction $(\mathrm{c} / \mathrm{r})$ for RdRp gene, and $5.4 \mathrm{c} / \mathrm{r}$ for Egene; ${ }^{* *}$ spike and nucleocapsid antibody; MxA-staining defined as high ( $>50 \%$ cells with intermediate to strong positive staining), or low ( $<50 \%)$. c, Confocal microscopy images of representative COVID-19 lung section stained for CD31 (green) and p-STING (red). Arrow indicates an endothelial cell with activated STING. d, Proportions of $\mathrm{CD}_{163}{ }^{+}$ macrophages and $\mathrm{CD} 1^{+}$endothelial cells among $\mathrm{p}$-STING ${ }^{+}$cells in COVID-19 lungs $(n=4)$. 
a

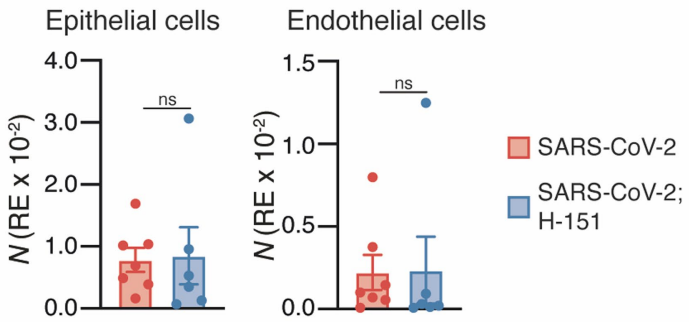

b

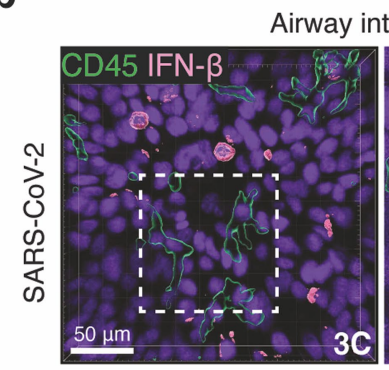

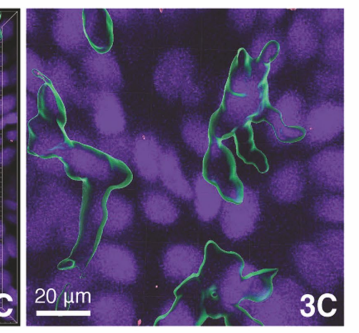

C

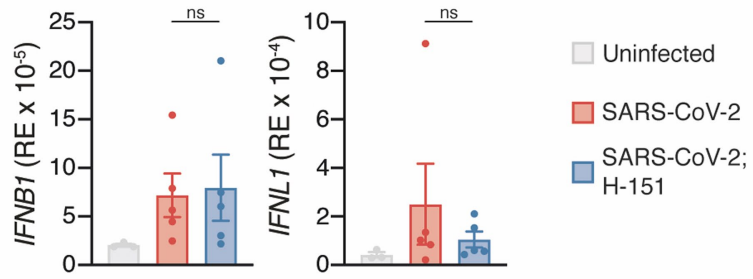

d
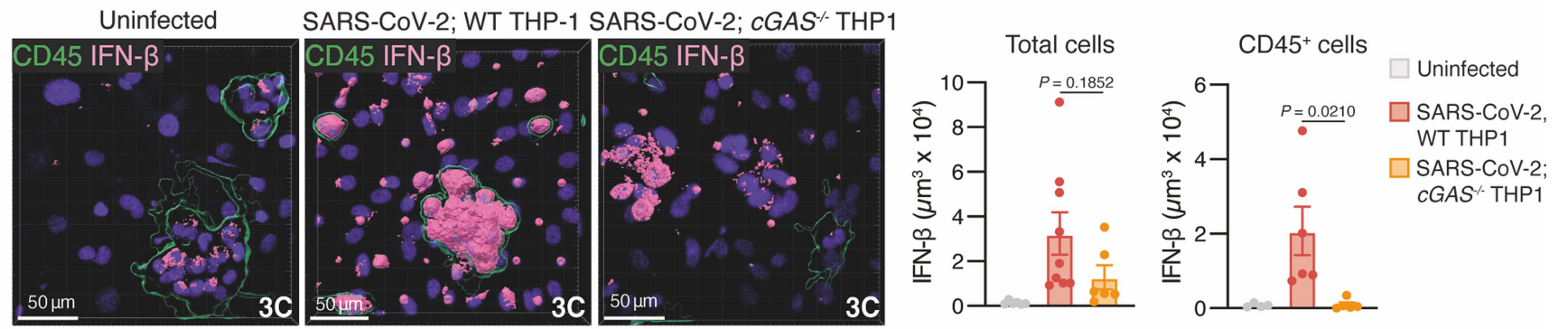

e
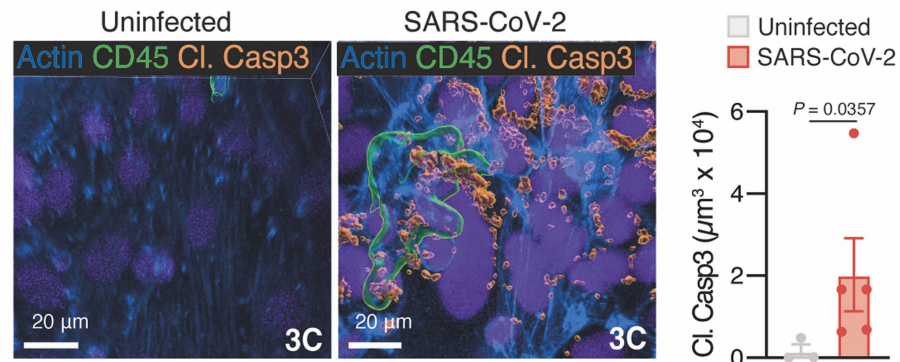

Extended Data Fig. 6 | Responses from distinct cell types after SARS-CoV-2 infection in the LoC system. a, mRNA expression levels of SARS-CoV- $2 \mathrm{~N}$ gene in epithelial and endothelial cells at $3 \mathrm{dpi}$ in 2-cell component LoCs. b, Representative 3D views of the airway surface of a LoC infected with SARS-CoV- 2 at $3 \mathrm{dpi}$. Areas with high levels of IFN $\beta$ (bright pink) are shown as surfaces. Macrophage surfaces are depicted in green, and nuclear labelling in purple. $\mathbf{c}, \mathrm{mRNA}$ expression levels of indicated genes in the epithelial cells at 3 dpi in uninfected $(n=3)$, infected $(n=5)$, and H-151-treated $(n=5)$ 2-cell component LoCs.d, Representative 3D views of the vascular face from uninfected and infected LoCs; PMA-activated WT and $c G A S^{-1-}$ THP-1 cells were added to vascular layer 2 days after infection. LoCs were analysed at $3 \mathrm{dpi}$ by quantifying the total volume with high IFN $\beta$ expression/volume with high IFN $\beta$ expression within macrophages from uninfected ( $n=6 / n=4$ fields of view), infected chips with WT THP- 1 cells added $(n=9 / n=6$ fields of view) and infected chips with $c G A S^{-1-}$ THP-1 cells added ( $n=6 / n=5$ fields of view) across $n=2$ LoCs in each case. e, Representative 3D views of the vascular face from uninfected and infected 3-component LoCs; volumes with high levels of cleaved caspase-3 (amber) are shown as surfaces. Quantification of the total volume with high cleaved caspase- 3 expression from uninfected $(n=3$ fields of view) and infected ( $n=5$ fields of view) from $n=1 \mathrm{LoC}$ in each case. ' $3 \mathrm{C}$ ' refers to 3-cell component (epithelial cells, endothelial cells, and macrophages) LoCs. Bars represent mean \pm s.e.m.; $P$ values were calculated by a two-tailed MannWhitney test (a) or one-way ANOVA followed by Tukey's multiple comparison tests $(\mathbf{c}, \mathbf{d})$. 
a shCtrl - endothelial, shCtrl - epithelial
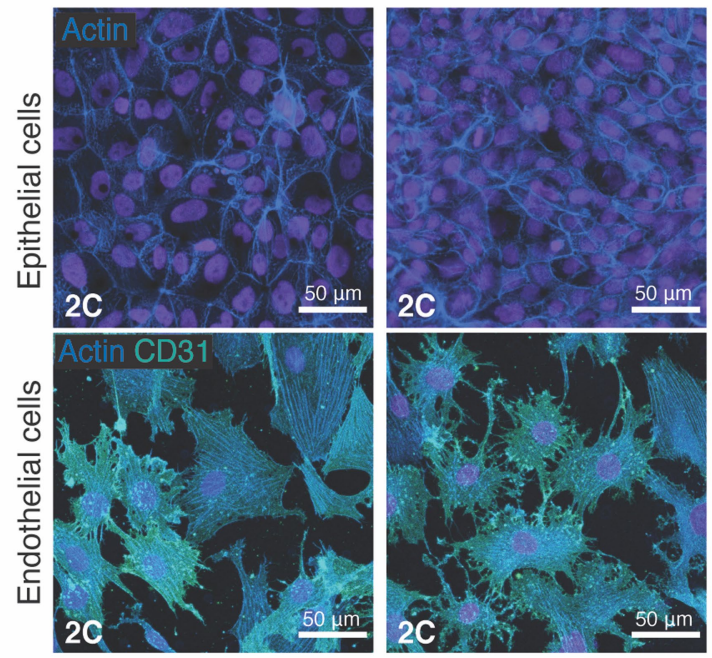

Epithelial Cells Endothelial Cells

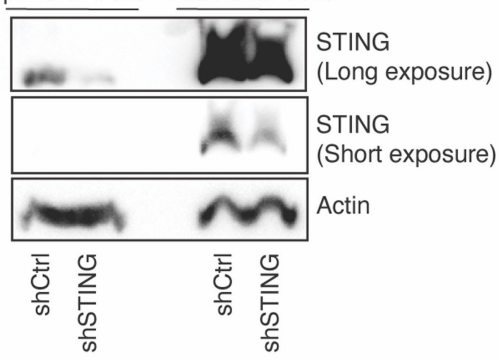

b
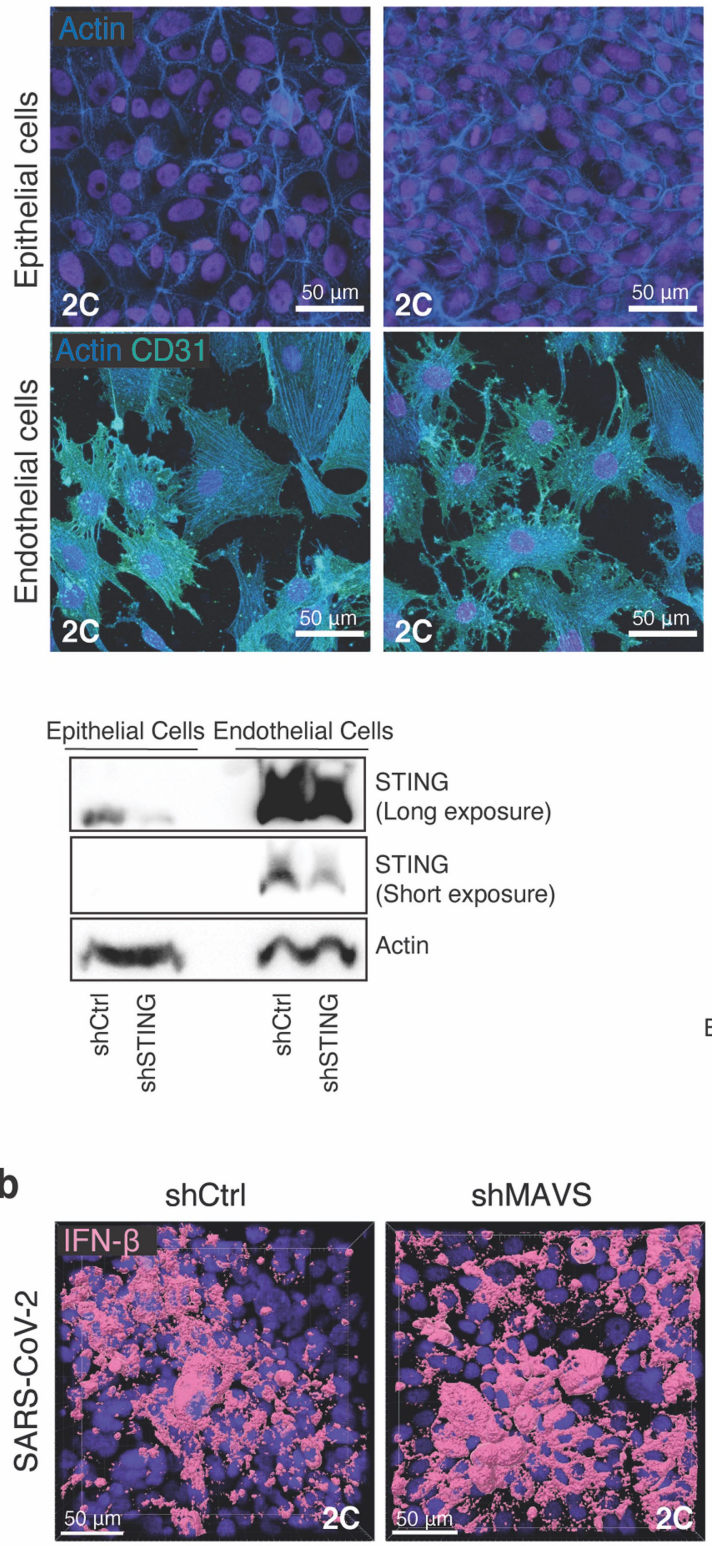

shSTING - endothelial, shSTING - epithelial
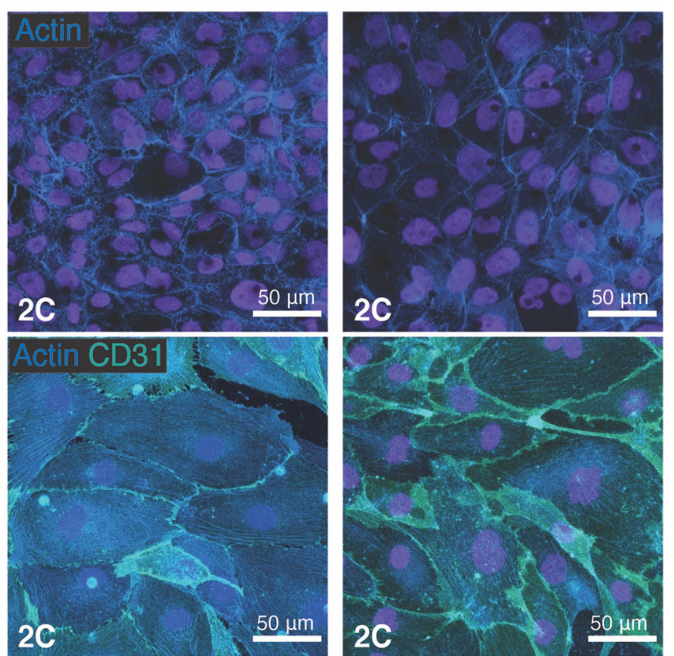

shSTING - endothelial,

shCtrl - epithelial

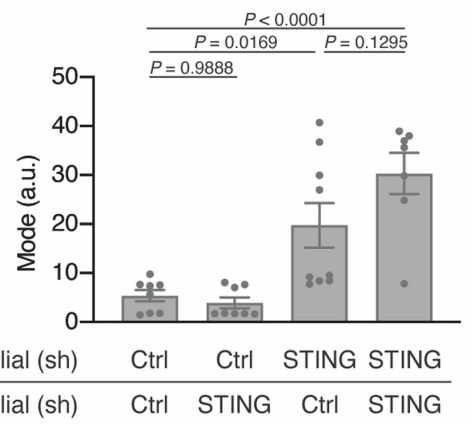

$$
\begin{aligned}
& \text { SARS-CoV-2; } \quad \square \text { SARS-CoV-2; } \\
& \text { shCtrl }
\end{aligned}
$$

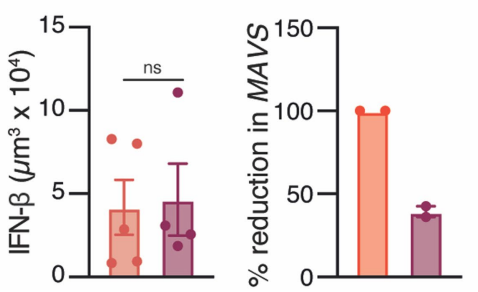

Extended Data Fig. 7 | Effect of innate immune sensors on endothelial cell response after SARS-CoV-2 infection in the LoC model. a, Representative images of the epithelial and endothelial cells on LoC infected with SARS-CoV-2 at $3 \mathrm{dpi}$ (above). Western blot characterization of STING expression in epithelial and endothelial cells treated with control (ctrl) and STING shRNAs (below, left). Modal value of CD31 expression in the endothelial layer of LoCs reconstituted with control or STING shRNA treated epithelial or endothelial cells (below, right). Each data point was calculated from a maximum intensity projection of CD31 expression from $n=7$ (sh Ctrl - epithelial/sh STING endothelial), $n=8$ (sh Ctrl - epithelial/sh Ctrl - endothelial and sh STING epithelial/sh Ctrl - endothelial) and $n=9$ (sh STING - epithelial/sh STING $=$ endothelial) fields of view from $n=1 \mathrm{LoC}$ in each case. $\mathbf{b}$, Representative 3D views at $3 \mathrm{dpi}$ of the vascular surface of a LoCs reconstituted with endothelial cells treated with ctrl or MAVS shRNA and infected with SARS-CoV-2. Volumes with high levels of IFN $\beta$ (bright pink) are shown as surfaces. Quantification of the volume with high IFN $\beta$ expression from $\operatorname{ctrl}(n=5$ fields of view) and MAVS shRNA ( $n=4$ fields of view) from $n=1$ LoC in each case. Reduction of MAVS mRNA in endothelial cells after shRNA transduction (right). '2C' refers to 2-cell component (epithelial cells, endothelial cells) LoCs. Bars represent mean \pm s.e.m.; $P$ values were calculated by a one-way ANOVA test followed by Tukey multiple comparison tests (a) or a two-tailed Mann-Whitney test (b). For gel source data, see Supplementary Fig.1. 


\section{a}

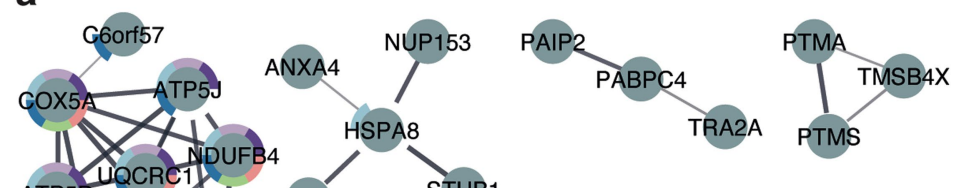

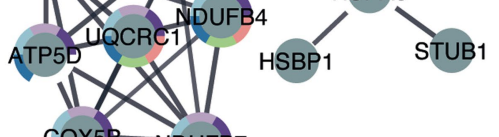

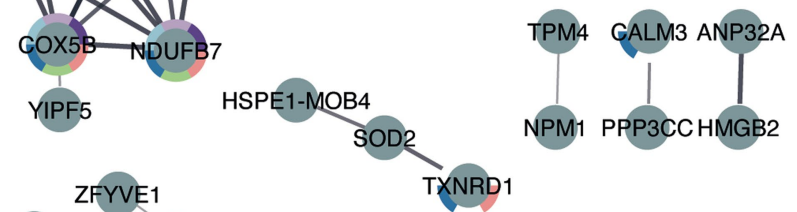

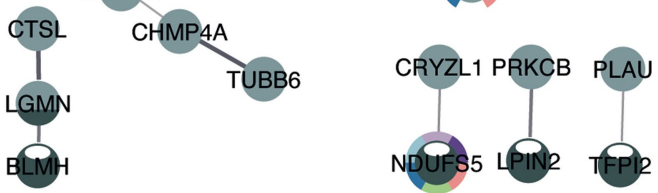

b

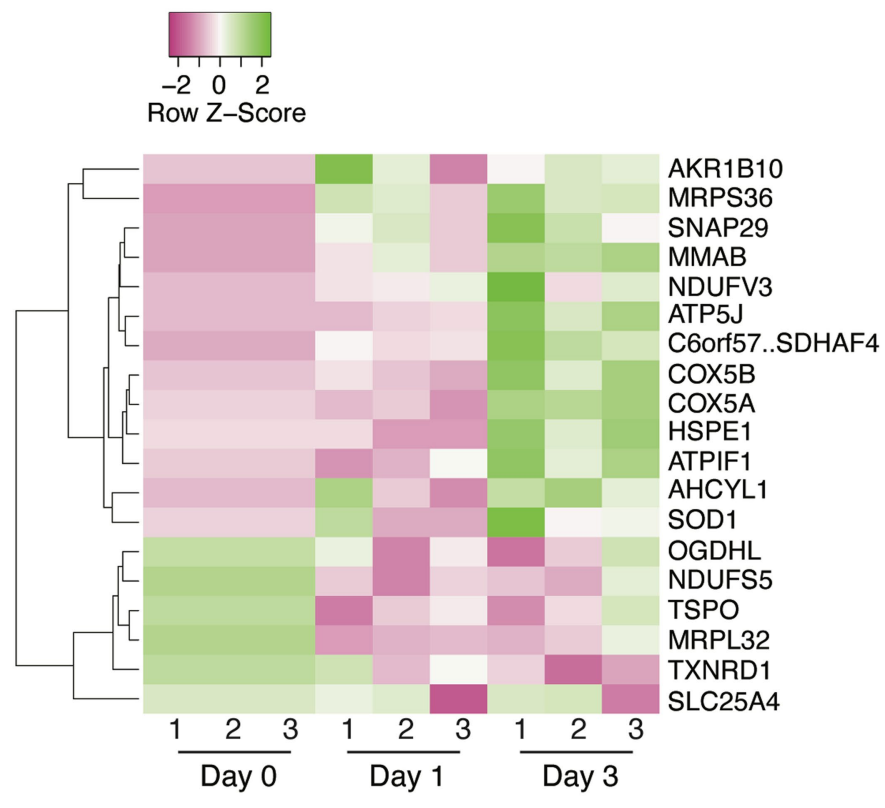

Electron transport chain

Oxidative phosphorylation

Thermogenesis

ATP metabolic process

Generation of precursor metabolites

Mitochondrial ATP coupled electron transport

C

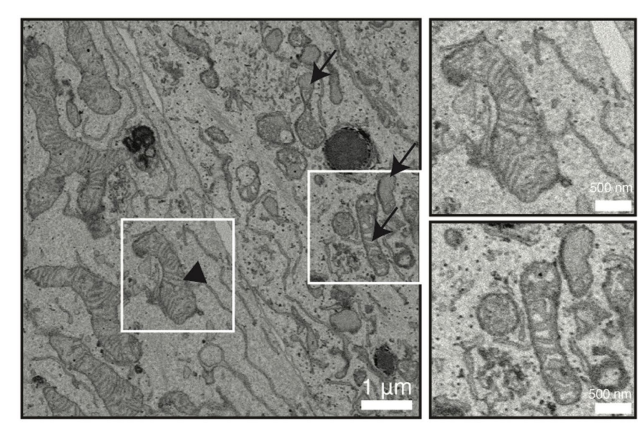

d

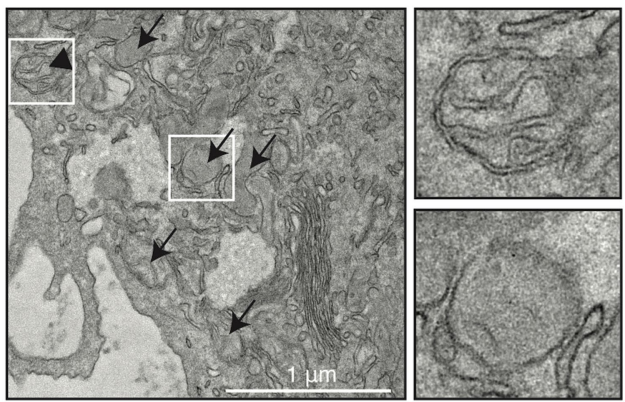

e
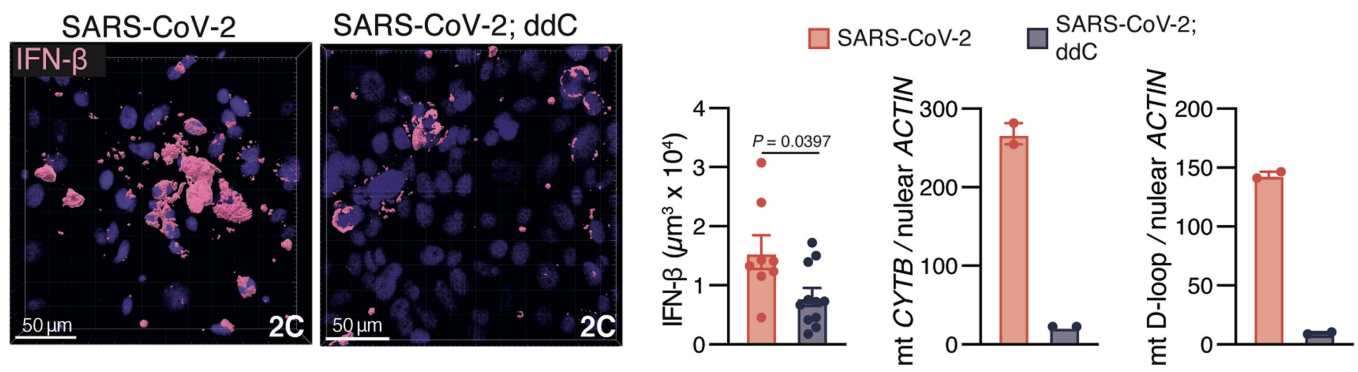

Extended Data Fig. 8 |See next page for caption. 


\section{Article}

Extended Data Fig. 8 | Disruption of mitochondrial homeostasis in endothelial cells after SARS-CoV-2 infection. a, Functional interactions between proteins with significantly altered expression identified by a pairwise analysis depicted via an interaction network generated from StringDB and clustered with the MCL algorithm. Functional annotations (GO Biological Processes/KEGG pathways) relevant to mitochondrial function are indicated. b, Heat map of data from mitochondrial proteins with significantly altered expression identified via a time-course analysis.c, d, Serial block scanning electron microscope image of endothelial cells in LoC system (c) or transmission electron microscopy image of endothelial cells in COVID-19 skin lesion (d). Arrows indicate damaged mitochondria with loss of cristae morphology with a magnified inset at bottom right. As control, arrowhead indicates an intact mitochondrium with a magnified inset at top right.

e, Representative 3D views at 3 dpi of the vascular surface of a LoCs reconstituted with endothelial cells treated or untreated with $20 \mu \mathrm{M} \mathrm{ddC}$ for 7 days in total and infected with SARS-CoV-2. Total mtDNA in endothelial cells was quantified by qPCR (right). Volumes with high levels of IFN $\beta$ (bright pink) are shown as surfaces. Quantification of the volume with high IFN $\beta$ expression from infected ( $n=11$ fields of view) and ddC treated infected $(n=8$ fields of view) from $n=1$ and $n=2$ LoCs respectively. '2C' refers to 2-cell component (epithelial cells, endothelial cells) LoCs. Bars represent mean \pm s.e.m.; $P$ values were calculated by a two-tailed Mann-Whitney test (e). 

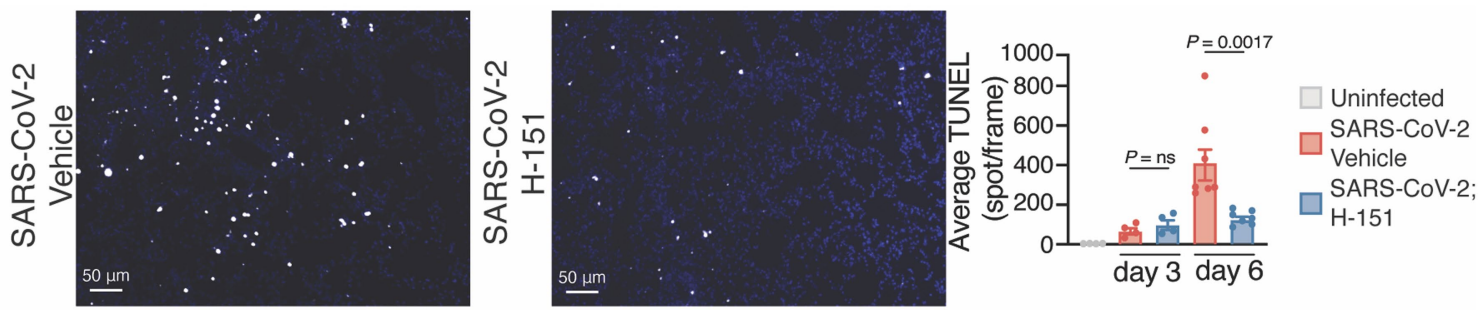

b

Lungs 6 dpi
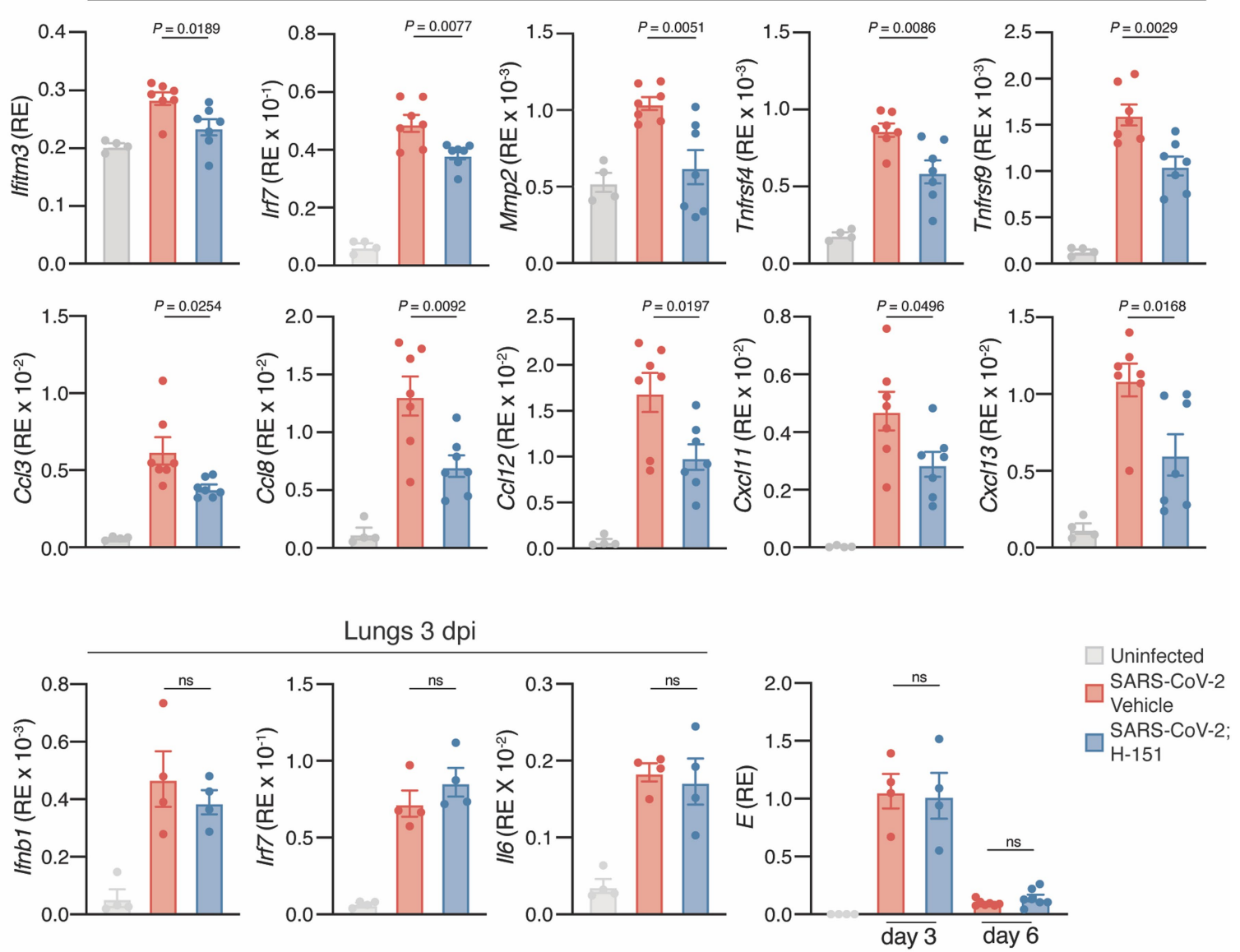

C

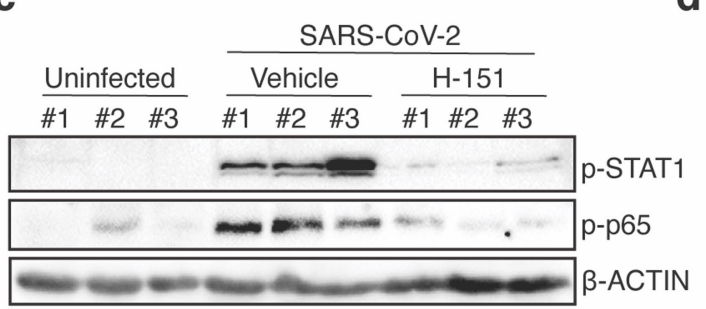

Extended Data Fig. 9|Prophylactic STING inhibition reduces pathology and inflammatory gene expression in the late stages of SARS-CoV-2 infection. a-d, Mice were infected with SARS-CoV-2 infection (intranasal; $1 \times 10^{4} \mathrm{PFU} /$ mouse) and intraperitoneal administration of vehicle or $\mathrm{H}-151$ was started at 1 day prior to infection. TUNEL assay performed on the infected lung sections collected at 3 or $6 \mathrm{dpi}$ is shown (a). mRNA levels of indicated genes isolated from uninfected and infected mouse lungs at $6 \mathrm{dpi}$ were analysed by

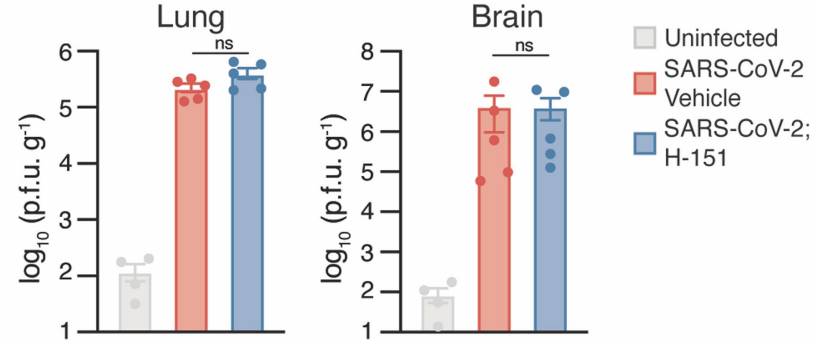

RT-qPCR (b). Tissue lysates from the lungs were subjected to Western blotting (c). Viral burden in the lungs and brains was analysed at $6 \mathrm{dpi}$ by plaque assay for infectious virus (d). Numbers are for $\mathbf{a}$ and $\mathbf{b}$ uninfected $(n=4)$, day 3 samples $(n=4)$, day 6 samples $(n=7)$; for d, uninfected $(n=4)$ and infected $(n=5)$. Throughout the figure, bars represent mean \pm s.e.m.; $P$ values were calculated by one-way ANOVA followed by Tukey multiple comparison tests. For gel source data, see Supplementary Fig. 1. 
a
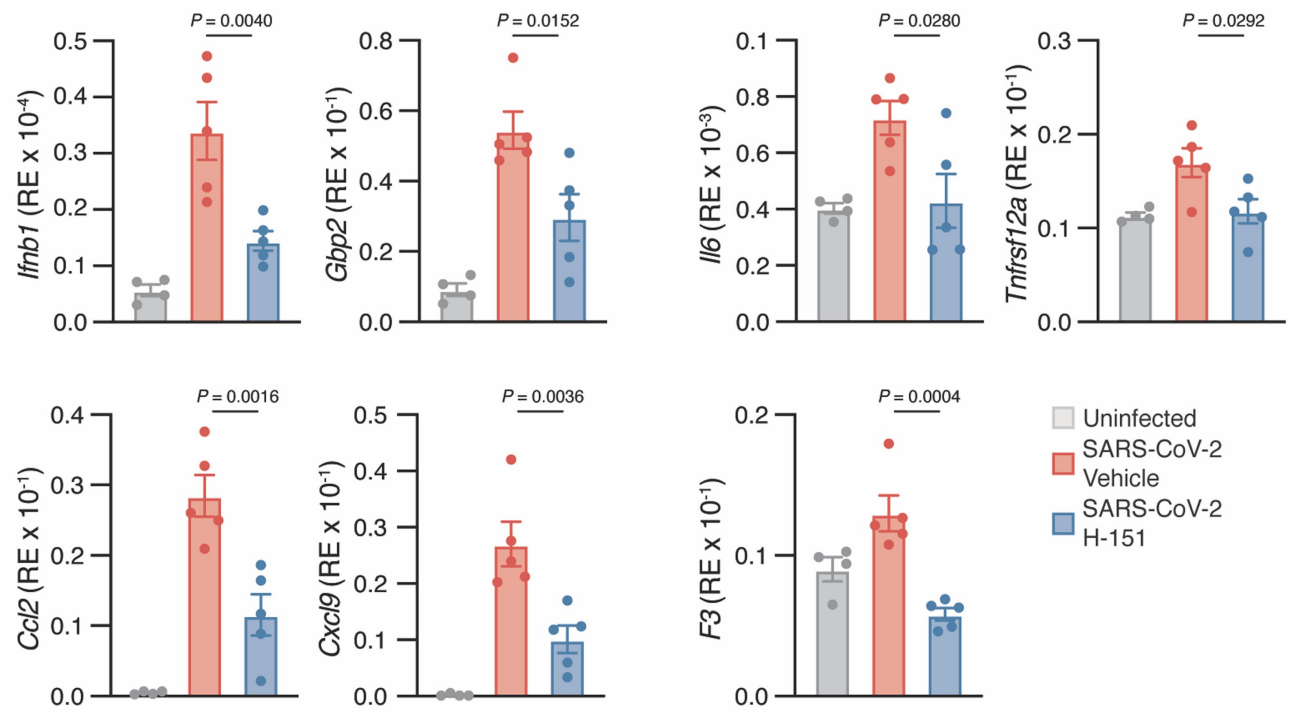

b
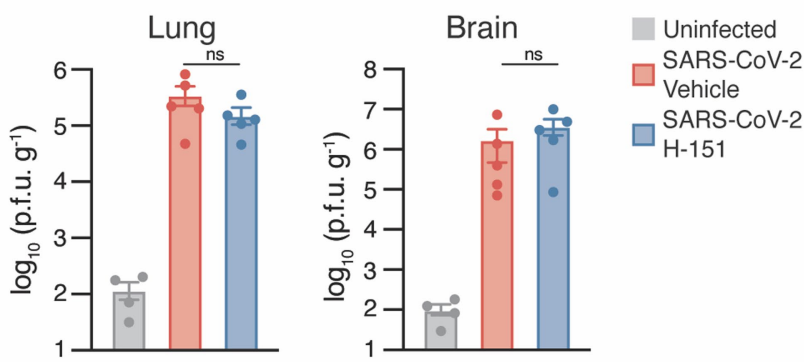

\section{C}

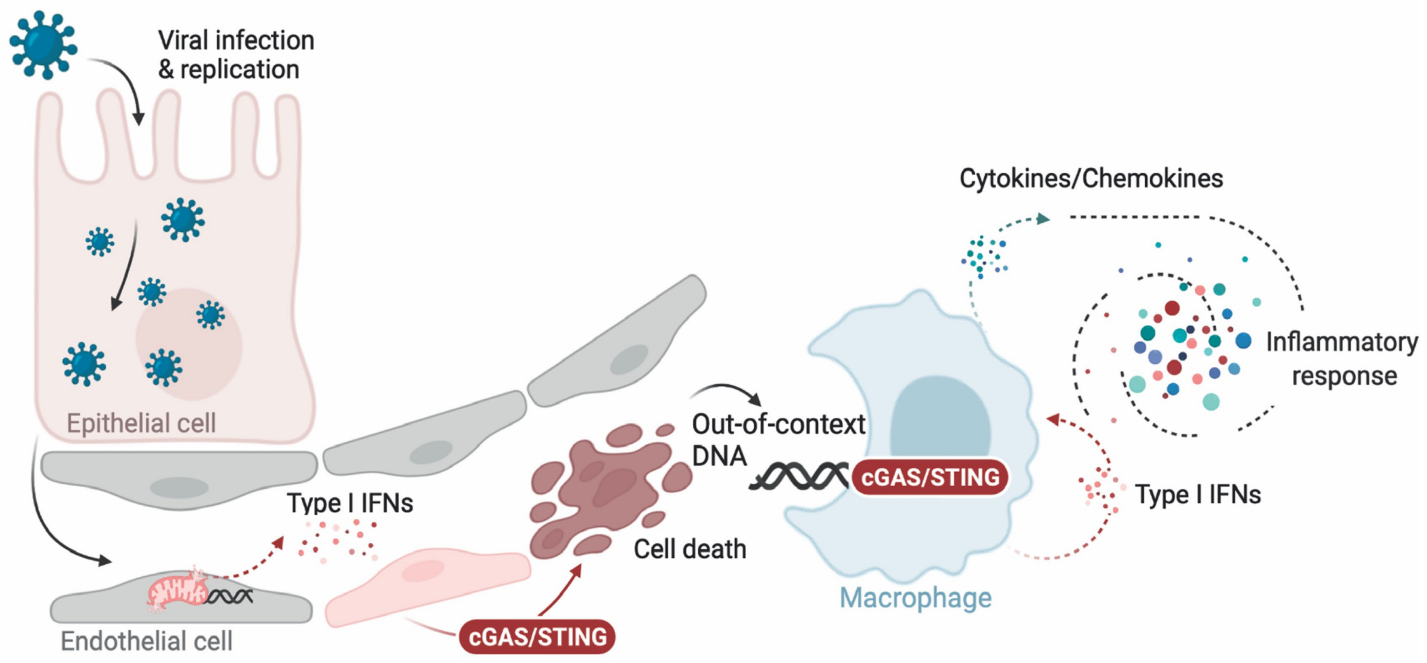

Extended Data Fig. 10 | Therapeutic inhibition of STING reduces pathology and inflammatory gene expression after SARS-CoV-2 infection. a, b, Mice were infected with SARS-CoV- 2 infection (intranasal; $1 \times 10^{4} \mathrm{PFU}$ per mouse) and intraperitoneal administration of vehicle or $\mathrm{H}-151$ was started at $2 \mathrm{dpi}$. mRNA was isolated from uninfected and infected mouse lungs and relative expression of indicated genes were analysed by RT-qPCR (a). Viral burden in the lungs and brains was analysed at 6 dpi by plaque assay for infectious virus (b).c, Model of the involvement of the cGAS-STING pathway in severe SARS-CoV-2 infection created with biorender.com. Numbers are uninfected $(n=4)$, infected $(n=5)$ $(\mathbf{a}, \mathbf{b})$. Throughout the figure, bars represent mean \pm s.e.m.; $P$ values were calculated by one-way ANOVA followed by Tukey multiple comparison tests. 


\section{Reporting Summary}

Nature Research wishes to improve the reproducibility of the work that we publish. This form provides structure for consistency and transparency in reporting. For further information on Nature Research policies, see our Editorial Policies and the Editorial Policy Checklist.

\section{Statistics}

For all statistical analyses, confirm that the following items are present in the figure legend, table legend, main text, or Methods section.

n/a Confirmed

$\square$ The exact sample size $(n)$ for each experimental group/condition, given as a discrete number and unit of measurement

$\square$ \ A statement on whether measurements were taken from distinct samples or whether the same sample was measured repeatedly

The statistical test(s) used AND whether they are one- or two-sided

$\square$ Only common tests should be described solely by name; describe more complex techniques in the Methods section.

\ $\square$ A description of all covariates tested

$\square$ \A description of any assumptions or corrections, such as tests of normality and adjustment for multiple comparisons

$\square$ A full description of the statistical parameters including central tendency (e.g. means) or other basic estimates (e.g. regression coefficient)

$\bigotimes$ AND variation (e.g. standard deviation) or associated estimates of uncertainty (e.g. confidence intervals)

$\varnothing$ For null hypothesis testing, the test statistic (e.g. $F, t, r$ ) with confidence intervals, effect sizes, degrees of freedom and $P$ value noted

Give $P$ values as exact values whenever suitable.

Х $\square$ For Bayesian analysis, information on the choice of priors and Markov chain Monte Carlo settings

Х $\square$ For hierarchical and complex designs, identification of the appropriate level for tests and full reporting of outcomes

\ $\square$ Estimates of effect sizes (e.g. Cohen's $d$, Pearson's $r$ ), indicating how they were calculated

Our web collection on statistics for biologists contains articles on many of the points above.

\section{Software and code}

Policy information about availability of computer code

Data collection Human Samples: Data were collected in part with nCounter ${ }^{\circledR}$ Systems, CaseCenter ${ }^{\mathrm{TM}}$ slide management system, ZEN 3.2 Imaging Software, Skanlt' ${ }^{\text {TM }}$ Software, WinTEM software

For Loc studies: Leica Application Suite Advanced Fluorescence for the Leica SP8 systems. T-qPCR data were acquired using the ABI Systems 7900HT Real-Time PCR system (Applied Biosciences)

For the rest: Western blots were acquired using Image Lab software (Biorad). RT-qPCR data were acquired using QuantStudio (5, 6 or 7) RealTime PCR system (ThermoFisher). Microscopic images were aquired using Axiovision software using Axioplan (Zeiss), Olympus OlyVIA software using Olympus V120 whole slide scanner. Western Blot images were analysed using Image Lab (Biorad). ECL signal was recorded using ChemiDoc XRS Biorad Imager.

Data analysis For Human Samples: Data were anlyzed using nSolver ${ }^{\mathrm{TM}}$ Analysis Software, ROSALIND ${ }^{\circledR}$ by OnRamp Bio, QuantCenter plugin 2.2 of Caseviewer 2.4 software, ZEN 3.2 Imaging Software, ImageJ 1.53c software, GraphPad PRISM 8, Microsoft Excel

Images in Loc studies were analysed and rendered using FIJI with Image J 1.53, and Bitplane Imaris 9.7.2. Data on proteomic networks from String DB was visualised using Cytoscape (3.8.2), and heatmaps generated using Heatmapper. 3D reconstructions of mitochondria were rendered using Blender 2.9. 
Policy information about availability of data

All manuscripts must include a data availability statement. This statement should provide the following information, where applicable:

- Accession codes, unique identifiers, or web links for publicly available datasets

- A list of figures that have associated raw data

- A description of any restrictions on data availability

Full scans for all western blots and the in-gel fluorescence images, are provided in Supplementary Fig. 1 and the Limma and Timecourse analysis of the proteomics data is provided in Supplementary Table 3. Source data for each figure are provided in the corresponding "Source Data" files. Raw data supporting the findings of this study are deposited at Zenodo and will be publicly available at doi:10.5281/zenodo.5818157 [available upon publication]. The proteomics dataset generated during this study is deposited in PRIDE with accession code [available during proof stage]. The genomics dataset generated during this study is deposited at GEO with accession code [available during proof stage].

\section{Field-specific reporting}

Please select the one below that is the best fit for your research. If you are not sure, read the appropriate sections before making your selection. Х Life sciences Behavioural \& social sciences Ecological, evolutionary \& environmental sciences

For a reference copy of the document with all sections, see nature.com/documents/nr-reporting-summary-flat.pdf

\section{Life sciences study design}

All studies must disclose on these points even when the disclosure is negative.

Sample size We have used at least three biological replicates for each experiment - unless stated otherwise. This is consistent with previous studies and accounts for biological variability in between distinct samples from inbred mice or from cell lines. The number of the mice used for the study was estimated based on the published studies related to this animal model. ( $n=5-8$ mice for histopathological studies and $n=14-15$ mice for the survival study. PMID: 32380511, PMID: 33257679)

Data exclusions No data was excluded.

Replication Experimental findings were reliably reproduced. The number $(n)$ of biological replicates or animals is indicated as an exact number in the figure legends.

Randomization In LoC experiments, for comparisons between different experimental conditions, fields of view ca. $232 \times 232 \times 10 \mu \mathrm{m} 3$ were chosen randomly from the epithelial and endothelial layers after a scan through the entire chip.

Mice were randomly allocated to distinct groups

Blinding $\quad$ LoC experiments: blinding was not possible as experimental conditions were evident from image data.

In vivo studies: Experimenters were blinded for the analysis of the histopathological scores for experimental groups.

\section{Reporting for specific materials, systems and methods}

We require information from authors about some types of materials, experimental systems and methods used in many studies. Here, indicate whether each material, system or method listed is relevant to your study. If you are not sure if a list item applies to your research, read the appropriate section before selecting a response.

\begin{tabular}{l|l} 
Materials \& experimental systems \\
\hline $\mathrm{n} / \mathrm{a}$ & Involved in the study \\
$\square$ & $\bigotimes$ Antibodies \\
$\square$ & $\bigotimes$ Eukaryotic cell lines \\
$\square$ & $\square$ Animaeontology and archaeology \\
$\square$ & $\square$ Human research participants \\
$\square$ & $\square$ Dual use research of concern
\end{tabular}

\begin{tabular}{l|l}
\multicolumn{2}{l}{ Methods } \\
\hline n/a & Involved in the study \\
$\searrow$ & $\square$ ChIP-seq \\
$\searrow$ & $\square$ Flow cytometry \\
$\searrow$ & $\square$ MRI-based neuroimaging
\end{tabular}

Antibodies

Antibodies used

Primary antibodies:

$\beta$-actin-HRP (C4, Santa Cruz 1:5000 dilution WB) 
Rabbit anti-Phospho-Stat1 (D4A7, Cell Signaling 1:1000 WB)

Rabbit anti-Phospho-p65 (S468) (Cell Signaling 1:1000 WB)

Mouse anti-human CD31 (P2B1, monoclonal, Abcam ab24590)

Mouse anti-human CD45 (MEM-28, monoclonal, Abcam ab8216)

Mouse anti-human CD45 (2D1, monoclonal, BioLegend 368537)

Mouse anti-human CD163 (10D6, DIAGNOSTIC BIOSYSTEMS, 1:50)

Rabbit anti-human CD163 (Cat\# LS-A10716, LSBio, 1:400)

Mouse anti-human CD123 (7G3, BD biosciences, 1:200)

Rabbit anti-human Myeloperoxidase (Cat\# A0398, DAKO, 1:1000)

Rabbit anti-human CD3 (2GV6, VENTANA, Ready to use)

Mouse anti-human CD31 (JC70A, DAKO, 1:100)

Rabbit anti-human IFN-beta (Cat\# PA5-20390, ThermoFisherScientific, 1:1000)

Rabbit anti-human Cleaved Caspase-3 (Asp175) (Cat \#9661, Cell Signaling Technology, 1:200)

Rabbit anti-human Phospho-STING (Ser366) (D7C3S, Cell Signaling Technology, 1:100)

Goat anti-human Mx1/2/3 (D-14, Santa Cruz, 1:50)

Secondary antibodies:

Donkey anti-rabbit IgG (H+L) AF546 (A10040, ThermoFisher, 1:500),

Goat anti-Rabbit IgG (H+L) AF546 (A11035, ThermoFisher, 1:500),

Goat anti-mouse IgG1 AF488 (A21121, ThermoFisher, 1:500),

Donkey anti-rabbit IgG (H+L) AF488 (A32790, ThermoFisher, 1:500),

Donkey Anti-Goat IgG H\&L (HRP) (ab97110, Abcam, 1:500),

Goat anti-mouse Alexa Fluor 488 (A-11029, Thermo Fisher),

Donkey anti-mouse Alexa Fluor 568 (A-10037, Thermo Fisher)

Donkey anti-rabbit Alexa Fluor 488 (A-21206, Thermo Fisher),

Donkey anti-rabbit Alexa Fluor 568 (A-10042, Thermo Fisher),

Validation

Primary Antibodies were validated for use for immunofluorescence for human or mouse respectively by the manufacturers as stated on their respective websites. Aliquots of secondary antibodies were provided by the Histology Core Facility at EPFL and have been validated by the manufacturers.

For patient samples:

Mouse anti-human CD163: IHC

Rabbit anti-human CD163: IHC

Mouse anti-human CD123: IF, IP, WB, Blocking, Neutralization

Rabbit anti-human Myeloperoxidase: IHC

Rabbit anti-human CD3: IHC

Mouse anti-human CD31: IHC

Rabbit anti-human IFN-beta: WB, IHC, IF, F, Elisa

Rabbit anti-human Cleaved Caspase-3 (Asp175): WB, IP, IHC, IF, F

Rabbit anti-human Phospho-STING (Ser366): WB

Goat anti-human $\mathrm{M} \times 1 / 2 / 3$ : WB, IP, IF, Elisa

\section{Eukaryotic cell lines}

Policy information about cell lines

Cell line source(s)

Primary human alveolar epithelial cells and human lung microvascular endothelial cells were purchased from Cell Biologics, USA via PeloBiosciences in Switzerland. WT and cGAS-/- THP-1 cells were purchased from Invivogen. Vero-E6 cells were a kind gift from the lab of Prof Carolyn Tapparel at the University of Geneva and HEK-293T cells were a gift from the lab of Prof Didier Trono at EPFL.

Authentication

The identity of primary human cells and of the THP-1 cell lines were verified by the supplier Cell Biologics and Invivogen respectively.

Mycoplasma contamination

All primary cells used without passage were verified to be mycoplasma free by the supplier. Passaged lung microvascular endothelial cells, THP-1, Vero-E6, and HEK-293T cell lines were verified to be mycoplasma free at EPFL.

Commonly misidentified lines

(See ICLAC register)

none

\section{Animals and other organisms}

Policy information about studies involving animals; ARRIVE guidelines recommended for reporting animal research

Laboratory animals

In this study, the following mouse strain was used B6.Cg-Tg(K18-ACE2)2PrImn/J (https://www.jax.org/strain/034860). For viral challenge female eight-week old mice were challenged intranasally with SARS-CoV-2.

Mice were housed in groups of up to 5 mice/cage at 18 degrees C-24 degrees $C$ ambient temperatures with $40-60 \%$ humidity. Mice were maintained on a 12 hour light/ dark cycle 6 am to $6 \mathrm{pm}$. Food and water were available ad libitum.

Wild animals

The study did not involve wild animals 
Field-collected samples The study did not involve samples collected from the field

Ethics oversight Animal experiments were approved by the Service de la Consommation et des Affaires Vétérinaires of the canton of Vaud (Switzerland) and were performed in accordance with the respective legal regulations.

Note that full information on the approval of the study protocol must also be provided in the manuscript.

\section{Human research participants}

Policy information about studies involving human research participants

Population characteristics

Patient_code gender age diagnosis

HD-01 M 48 healthy donor

HD-02 M 55 healthy donor

HD-03 M 30 healthy donor

HD-04 M 44 healthy donor

PV-01 M 21 plaque-type psoriasis

PV-02 M 44 plaque-type psoriasis

PV-03 M 66 plaque-type psoriasis

PV-04 M 49 plaque-type psoriasis

PV-05 M 41 plaque-type psoriasis

PV-06 F 47 plaque-type psoriasis

PV-07 M 57 plaque-type psoriasis

PV-08 M 43 plaque-type psoriasis

PV-09 M 29 plaque-type psoriasis

PV-10 M 32 plaque-type psoriasis

PV-11 M 34 plaque-type psoriasis

PV-12 M 33 plaque-type psoriasis

PV-13 F 37 plaque-type psoriasis

PV-14 M 66 plaque-type psoriasis

PV-15 M 39 plaque-type psoriasis

PV-16 F 48 plaque-type psoriasis

PV-17 F 55 plaque-type psoriasis

PV-18 M 63 plaque-type psoriasis

PV-19 M 48 plaque-type psoriasis

PV-20 M 58 plaque-type psoriasis

PV-21 M 36 plaque-type psoriasis

AD-01 M 33 atopic dermatitis

AD-02 M 32 atopic dermatitis

AD-03 F 23 atopic dermatitis

AD-04 M 39 atopic dermatitis

AD-05 M 31 atopic dermatitis

AD-06 F 22 atopic dermatitis

AD-07 M 53 atopic dermatitis

AD-08 M 28 atopic dermatitis

AD-09 F 29 atopic dermatitis

AD-10 M 25 atopic dermatitis

AD-11 M 82 atopic dermatitis

AD-12 M 27 atopic dermatitis

AD-13 M 44 atopic dermatitis

AD-14 M 27 atopic dermatitis

AD-15 M 61 atopic dermatitis

AD-16 M 46 atopic dermatitis

LP-01 M 33 lichen planus

LP-02 F 54 lichen planus

LP-03 M 39 lichen planus

LP-04 F 47 lichen planus

LP-05 M 57 lichen planus

CLE-01 F 40 cutaneous lupus erythematosus

CLE-02 M 40 cutaneous lupus erythematosus CLE-03 F 52 cutaneous lupus erythematosus

CLE-04 F 47 cutaneous lupus erythematosus

CLE-05 F 31 cutaneous lupus erythematosus

CLE-06 M 78 cutaneous lupus erythematosus

CLE-07 M 46 cutaneous lupus erythematosus

CLE-08 M 36 cutaneous lupus erythematosus

CLE-09 F 64 cutaneous lupus erythematosus

CLE-10 F 46 cutaneous lupus erythematosus

CLE-11 F 27 cutaneous lupus erythematosus

CLE-12 F 21 cutaneous lupus erythematosus

CLE-13 F 67 cutaneous lupus erythematosus

CLE-14 F 52 cutaneous lupus erythematosus

CLE-15 F 25 cutaneous lupus erythematosus

CLE-16 F 50 cutaneous lupus erythematosus

COVID-01 F 62 COVID-19-associated skin lesion 
COVID-02 F 65 COVID-19-associated skin lesion

COVID-03 F 34 COVID-19-associated skin lesion

COVID-04 F 21 COVID-19-associated skin lesion

COVID-05 F 62 COVID-19-associated skin lesion

COVID-06 M 47 COVID-19-associated skin lesion

COVID-07 M 18 COVID-19-associated skin lesion

COVID-08 M 22 COVID-19-associated skin lesion

COVID-09 M 56 COVID-19-associated skin lesion

COVID-10 M 80 COVID-19-associated skin lesion

\section{Recruitment}

Ethics oversight
Residual skin materials were obtained from the Dermatology Biobank at CHUV from patients with COVID-19-associated skin lesions, cutaneous lupus erythematosus (CLE), lichen planus (LP), atopic dermatitis (AD), and plaque-type psoriasis (PV). For healthy controls, residual healthy skin was obtained from surgery.

Studies were approved by the University Hospital of Lausanne (CHUV) and were performed in accordance with the guidelines of the Declaration of Helsinki and were reviewed by the ethical committee board of the canton of Vaud (CER-VD 2020-02204).

Note that full information on the approval of the study protocol must also be provided in the manuscript. 